\title{
Ambient metrics with exceptional holonomy
}

\author{
THOMAS LEISTNER AND PAWEŁ NUROWSKI
}

\begin{abstract}
We present conformal structures in signature $(3,2)$ for which the holonomy of the Fefferman-Graham ambient metric is equal to the non-compact exceptional Lie group $\mathrm{G}_{2(2)}$. We write down the resulting 8-parameter family of $\mathrm{G}_{2(2)}$-metrics in dimension seven explicitly in an appropriately chosen coordinate system on the ambient space.
\end{abstract}

Mathematics Subject Classification (2010): 53A30 (primary); 53B30, 53C29 (secondary).

\section{Introduction and the main result}

The holonomy group of a semi-Riemannian manifold $(M, g)$ at a point $p \in M$ is defined as the group of parallel transports along loops based at $p$. It provides a powerful tool to study the geometric structure of the manifold and, for example, enables to answer questions about the existence of parallel sections of geometric vector bundles. In his seminal paper [5], Berger gave a list of possible holonomy groups of simply connected (semi-)Riemannian manifolds under the assumption that the group acts irreducibly on the tangent space at $p$. The list comprised groups that only occur as holonomies in certain dimensions, such as the exceptional compact Lie group $\mathrm{G}_{2}$ as the holonomy group of a 7-dimensional Riemannian manifold, or its non-compact real form $\mathrm{G}_{2(2)} \subset \mathrm{SO}(4,3)$, as the holonomy group of a manifold with metric of signature $(4,3)$.

Since Berger's paper the question whether or not the exceptional groups on the list can be realised as holonomy groups of semi-Riemannian manifolds was studied with great interest. In Reference [6] Bryant proved that there exist semiRiemannian metrics with exceptional holonomy groups, in particular, with holonomy $\mathrm{G}_{2}$ and $\mathrm{G}_{2(2)}$. After this, in the Riemannian case, research was focussed on the construction of geodesically complete and compact metrics with exceptional holon-

This work was supported by the SFB 676 of the German Research Foundation and by the first author's Start-Up-Grant of the Faculty of Engineering, Computer and Mathematical Sciences of the University of Adelaide. The second author acknowledges the support from the Polish Ministry of Research and Higher Education under grants NN201 607540 and NN202 104838.

Received March 26, 2010; accepted January 13, 2011. 
omy. After the first complete examples by Bryant and Salamon [8], the compact case was finally settled by Joyce [21,22]. For the indefinite case, after the existence of metrics with holonomy $\mathrm{G}_{2(2)}$ was verified in Bryant's paper [6], Salamon observed [33, remark on page 168] that the construction method in [8] for Riemannian metrics can be modified to the indefinite case. Apart from this observation, so far only a few explicit examples of signature $(4,3)$-metrics with holonomy group $\mathrm{G}_{2(2)}$ have been constructed explicitly, e.g. in [12].

In the present article we will use the Fefferman-Graham ambient metric construction of conformal geometry and the conformal classes introduced in [31] in order to construct explicitly an 8-parameter family of metrics with holonomy equal to $\mathrm{G}_{2(2)}$. These conformal classes were shown to have the normal conformal Cartan connection reduced to a $\mathrm{G}_{2(2)}$-Cartan connection. Our aim here is to show that, generically, the Fefferman-Graham ambient metrics for these conformal classes constitute explicit examples of signature $(4,3)$-metrics with full $\mathrm{G}_{2(2)}$ holonomy.

Our main tool is the Fefferman-Graham ambient metric construction for a conformal manifold $(M,[g])$. This is a Ricci-flat metric on a neighbourhood $\widetilde{M}$ in $\mathcal{C} \times \mathbb{R}$ of the cone

$$
\mathcal{C}=\left\{\left(g_{p}, p\right) \mid p \in M, g \in[g]\right\},
$$

which encodes the conformal structure of $(M,[g])$. In odd dimensions, this metric always exists, is unique, and provides a way of describing a conformal structure invariantly by means of semi-Riemannian geometry. Every metric $g$ in the conformal class $[g]$ defines an embedding $M \ni p \mapsto\left(g_{p}, p\right) \in \mathcal{C}$ and thus an identification of $\widetilde{M}$ with $\mathbb{R}_{+} \times M \times \mathbb{R}$ via $(t, p, \rho) \mapsto\left(t^{2} g_{p}, \rho\right)$. Using this identification, the Fefferman-Graham ambient metric is given as a formal power series

$$
\widetilde{g}=2(t \mathrm{~d} \rho+\rho \mathrm{d} t) \mathrm{d} t+t^{2}\left(g+\sum_{k=1}^{\infty} \rho^{k} \mu_{k}\right)
$$

with certain symmetric $(2,0)$ tensors $\mu_{k}$ on $M$ that are determined by the condition that $\tilde{g}$ is a Ricci-flat metric. For example, the first two terms are given by

$$
\begin{aligned}
& \left(\mu_{1}\right)_{a b}=2 \mathrm{P}_{a b} \\
& \left(\mu_{2}\right)_{a b}=-B_{a b}+\mathrm{P}_{a k} \mathrm{P}_{b}^{k},
\end{aligned}
$$

where $\mathrm{P}_{a b}$ denotes the Schouten tensor of $g$ and $B_{a b}$ is the Bach tensor of $g$. When formulating our main result in Theorem 1.1 we will change the coordinate $\rho$ to $u:=-\rho t$, i.e. $\mathrm{d} u=-t \mathrm{~d} \rho-\rho \mathrm{d} t$ (see $[14,15]$ and our Section 2 for details).

The conformal class $\left[g_{F}\right]$ of $(3,2)$-signature metrics constructed by the second author in Reference [31] is defined on every 5-manifold $M$ equipped with a nonintegrable rank 2 distribution $S$ whose small growth vector is $(2,3,5)$. Such distributions in dimension five are called $(2,3,5)$-distributions. According to the classical results of Elie Cartan [11] and David Hilbert [20], (2, 3, 5)-distributions are in one to one correspondence with ODE's of the form

$$
z^{\prime}=F\left(x, y, y^{\prime}, y^{\prime \prime}, z\right), \quad F_{y^{\prime \prime} y^{\prime \prime}} \neq 0,
$$


for two real functions $y=y(x), z=z(x)$ of one real variable $x$. In particular, given $F$, the distribution is defined by

$$
S=\operatorname{Span}\left(\partial_{y^{\prime \prime}}, \partial_{x}+y^{\prime} \partial_{y}+y^{\prime \prime} \partial_{y^{\prime}}+F \partial_{z}\right),
$$

and the metrics $\left[g_{F}\right]$ are defined in terms of the function $F$ and its apropriate derivatives as in [31]. A construction of these metrics can also be found in [10].

By the construction the metrics $\left[g_{F}\right]$ have reduced conformal holonomy $H$. The group $H$ is contained in the noncompact exceptional Lie group $\mathrm{G}_{2(2)} \subset \mathrm{SO}(4,3)$ [31]. Moreover, it was recently shown in [19] that every conformal class $[g]$ of $(3,2)$ signature metrics, whose conformal holonomy $H$ is contained in $\mathrm{G}_{2(2)}$ must be locally conformally equivalent to one of the structures $\left[g_{F}\right]$.

Since metrics $\left[g_{F}\right]$ include all the conformal metrics with the exceptional conformal holonomy $\mathrm{G}_{2(2)}$, it is interesting to ask about the properties of their Fefferman-Graham ambient metrics $\widetilde{g}_{F}$. In Reference [32] properties of the ambient metric were studied for the conformal class of metrics $\left[g_{F}\right]$ with

$$
F=\left(y^{\prime \prime}\right)^{2}+a_{0}+a_{1} y^{\prime}+a_{2}\left(y^{\prime}\right)^{2}+a_{3}\left(y^{\prime}\right)^{3}+a_{4}\left(y^{\prime}\right)^{4}+a_{5}\left(y^{\prime}\right)^{5}+a_{6}\left(y^{\prime}\right)^{6}+b z,
$$

where $a_{\mu}, \mu=0, \ldots, 6$, and $b$ are real constants. The results about properties of $\left[g_{F}\right]$ with this $F$ from [32] are strengthened in the present paper. By setting $q:=y^{\prime \prime}$ and $p:=y^{\prime}$ we have

$$
F=q^{2}+\sum_{i=0}^{6} a_{i} p^{i}+b z
$$

and the conformal class $\left[g_{F}\right]$ is explicitly given by the metric

$$
g_{F}=2 \theta^{1} \theta^{5}-2 \theta^{2} \theta^{4}+\left(\theta^{3}\right)^{2}
$$

where the co-frame $\theta^{i}$ is given by

$$
\theta^{i}=\mathrm{e}^{-\frac{2 b}{3} x} \hat{\theta}^{i}
$$

with

$$
\begin{aligned}
& \hat{\theta}^{1}=\mathrm{d} y-p \mathrm{~d} x \\
& \hat{\theta}^{2}=\mathrm{d} z-F \mathrm{~d} x-2 q(\mathrm{~d} p-q \mathrm{~d} x) \\
& \hat{\theta}^{3}=-\frac{2^{4 / 3}}{\sqrt{3}}(\mathrm{~d} p-q \mathrm{~d} x) \\
& \hat{\theta}^{4}=2^{-1 / 3} \mathrm{~d} x \\
& \hat{\theta}^{5}=3 A_{2}(\mathrm{~d} y-p \mathrm{~d} x)+\frac{2^{2 / 3}}{3} b(\mathrm{~d} p-q \mathrm{~d} x)-2^{2 / 3} \mathrm{~d} q+A_{1} \mathrm{~d} x,
\end{aligned}
$$


where

$$
\begin{aligned}
& A_{1}=\frac{1}{2^{1 / 3}}\left(a_{1}+2 a_{2} p+3 a_{3} p^{2}+4 a_{4} p^{3}+5 a_{5} p^{4}+6 a_{6} p^{5}+2 b q\right), \\
& A_{2}=\frac{1}{45 \cdot 2^{2 / 3}}\left(9 a_{2}+27 a_{3} p+54 a_{4} p^{2}+90 a_{5} p^{3}+135 a_{6} p^{4}+2 b^{2}\right) .
\end{aligned}
$$

Our aim here is to prove the following result about the conformal classes defined by these metrics.

Theorem 1.1. Let $\left(M,\left[g_{F}\right]\right)$ be a conformal structure associated with a $(2,3,5)$ distribution defined by a function

$$
F=\left(y^{\prime \prime}\right)^{2}+a_{0}+a_{1} y^{\prime}+a_{2}\left(y^{\prime}\right)^{2}+a_{3}\left(y^{\prime}\right)^{3}+a_{4}\left(y^{\prime}\right)^{4}+a_{5}\left(y^{\prime}\right)^{5}+a_{6}\left(y^{\prime}\right)^{6}+b z,
$$

with $a_{\mu}, \mu=0, \ldots, 6$, and $b$ being real constants, i.e. given by the metric in equation (1.4). This 8-parameter family of conformal structures has the following properties:

(1) For each value of the parameters $a_{\mu}$ and $b$ there exists a metric $g_{F}$ in the class $\left[g_{F}\right]$ and ambient coordinates $(t, u)$, in which the Fefferman-Graham ambient metric $\tilde{g}_{F}$ for $g_{F}$ is given explicitely by

$$
\widetilde{g}_{F}=-2 \mathrm{~d} t \mathrm{~d} u+t^{2} g_{F}-2 t u \mathrm{P}-u^{2} B .
$$

Here $\mathrm{P}$ and $B$ are the respective Schouten and Bach tensors for $g_{F}$.

(2) If at least one of $a_{3}, a_{4}, a_{5}$ or $a_{6}$ is not zero, then the metric $\tilde{g}_{F}$ has the full exceptional group $\mathrm{G}_{2(2)}$ as its pseudo-Riemannian holonomy.

In the following proof of this theorem we will refer to statements that are proven in the paper.

Proof. When proving the theorem we fix the metric $g_{F} \in\left[g_{F}\right]$ given as in formula (1.4). In order to prove (1) of the theorem, recall that the first terms in the Fefferman-Graham ambient metric expansion are given by equations (1.1) and (1.2). Using the formulae for the Schouten tensor $\mathrm{P}$ and the Bach tensor $B$ of $g_{F}$ given in the appendix, it follows that $\mathrm{P}_{a k} \mathrm{P}_{b}^{k}=0$. Again, using the formulae in the appendix, one checks that the metric

$$
-2 \mathrm{~d} u \mathrm{~d} t+t^{2} g_{F}-2 t u \mathrm{P}-u^{2} B
$$

is Ricci flat. Since $g_{F}$ is real analytic, the uniqueness of the ambient metric in the analytic category in odd dimensions $[14,15]$ implies that this is the ambient metric $\tilde{g}_{F}$ for $\left[g_{F}\right]$. This proves (1) of the theorem.

Now we prove (2) of the theorem. Using the formulae for $g_{F}$ given in the appendix, one verifies that $\widetilde{g}_{F}$ admits a parallel spinor which is not null. In Proposition 4.1 we will give this spinor explicitly. Hence, since $\mathrm{G}_{2(2)}$ is the stabiliser of 
a non-null spinor, the holonomy $H$ of $\widetilde{g}_{F}$ is contained in $\mathrm{G}_{2(2)}$. Now we have to verify that $H$ is equal to $\mathrm{G}_{2(2)}$.

First note that the naive approach of calculating the curvature of $\widetilde{g}_{F}$ and of showing that it generates $\mathrm{G}_{2(2)}$ does not work since the curvature of $\widetilde{g}_{F}$ is highly degenerated. In the best case, the Riemann tensor of $\widetilde{g}_{F}$ mapping $\Lambda^{2} \mathbb{R}^{7}$ to $\mathrm{G}_{2(2)}$ has rank four. On the other hand, obtaining the full set of the first (or higher) derivatives of the curvature for the general $F$ from the theorem is beyond our calculational skills. Thus we have to use more subtle arguments. They are as follows:

In order to verify that $H$ is equal to $\mathrm{G}_{2(2)}$, first assume that $H$ acts irreducibly on $\mathbb{R}^{4,3}$. By Berger's list of irreducible holonomy groups of non-symmetric pseudoRiemannian manifolds [5], which contains only $\mathrm{G}_{2(2)}$ in dimension 7 (see also [4] for the corresponding list of groups admitting invariant spinors), $\widetilde{g}_{F}$ must be locally symmetric if $H \neq \mathrm{G}_{2(2)}$. This can be excluded by a direct calculation of derivatives of the curvature. For example, we verified by a direct calculation that $\widetilde{\nabla}_{1} \widetilde{R}_{1212} \neq 0$, where the indices refer to the orthonormal coframe $\xi^{0}, \ldots, \xi^{6}$ given on page 429 .

Hence, if $H$ is not equal to $\mathrm{G}_{2(2)}$, it must admit an invariant subspace $V \subset$ $\mathbb{R}^{4,3}$. The exclusion of this situation will be based on the following two pairs of statements. The first describes the relation between the geometry of the ambient metric and the existence of certain metrics in a conformal class. Let $(\widetilde{M}, \widetilde{g})$ be the ambient metric for a conformal class $[g]$. Then the following holds:

(A) If $(\tilde{M}, \widetilde{g})$ admits a parallel line bundle, then, on an open dense set of $M$, every metric in $[g]$ is locally conformal to an Einstein metric (see Theorem 2.2).

(B) If $(\tilde{M}, \widetilde{g})$ admits a parallel bundle of totally null 2-planes, then, on an open dense set of $M$, every metric in $[g]$ is locally conformal to a metric $g$ which admits a parallel null line bundle $L$ such that $\left.L^{\perp}\right\lrcorner \mathrm{Ric}^{g}=0$ (see Theorem 2.6).

The second pair of statements excludes the existence of certain metrics in the conformal class $\left[g_{F}\right]$ under assumptions on $F$. In Theorems 3.7 and 3.10 we prove: If at least one of the constants $a_{3}, a_{4}, a_{5}$ or $a_{6}$ is not equal to zero, then

(C) the class $\left[g_{F}\right]$ does not contain a local Einstein metric (see Theorem 3.7).

(D) the class $\left[g_{F}\right]$ does not contain a local metric $g$ that admits a $\nabla^{g}$-parallel null line $L$ and whose Ricci tensor is annihilated by $L^{\perp}$ (see Theorem 3.10).

Now we assume that the subspace $V$, which is invariant under the holonomy $H$ of $\widetilde{g}_{F}$, is non-degenerate, i.e. $V \cap V^{\perp}=\{0\}$. By the local version of the decomposition theorem by de Rham and $\mathrm{Wu}$ [34, Proposition 3] this implies that the ambient metric splits locally as a pseudo-Riemannian product metric, $\widetilde{g}_{F}=g_{1}+g_{2}$. Since $\widetilde{g}_{F}$ is Ricci-flat, both, $g_{1}$ and $g_{2}$ have to be Ricci-flat. Since one of them is a metric in dimension $\leq 3$, its Ricci-flatness implies that it is flat. In this case $\widetilde{g}_{F}$ would admit at least one parallel vector field. Now we use statement (A) that the existence of a parallel vector field for the ambient metric implies that, on a dense open set, $g_{F}$ is locally conformal to an Einstein metric. Under the assumptions on $F$, statement (C) gives the contradiction. Hence, $\widetilde{g}_{F}$ does not admit a non-degenerate invariant subspace under the holonomy representation. 
Now assume that the $H$-invariant vector space $V$ is degenerate, i.e. $W:=$ $V \cap V^{\perp}$ is a non-trivial totally null space. Then the dimension of $W$ has to be $\leq 3$. The first case is that $W$ is one dimensional, i.e. that the ambient metric admits a parallel null line bundle. Again by (A), $g_{F}$ must be locally conformally Ricci flat on a dense open set, which is again in contradiction to the statement (C).

Now, if $W$ is a null 2-plane, statement (B) shows that locally there is a metric $g \in\left[g_{F}\right]$ with a $\nabla^{g}$-parallel null line $L$ and with $\operatorname{Ric}^{g}(Y,)=$.0 for all $Y \in L^{\perp}$. By the assumptions on $F$ this contradicts the statement (D) that for the given $F$ 's this is not possible.

Finally, we assume that $W$ is maximally null, i.e. three-dimensional. In this case there exists a pure null spinor which scales under $H[23,25]$ and thus defines a line of spinors that is parallel for $\tilde{g}_{F}$. This means that we are in the situation where we have the parallel non-null spinor defining $\mathrm{G}_{2(2)}$ and a parallel null line of spinors. Now we use the fact (which is proven in Lemma 4.2) that in this situation there exists a parallel line of vectors for $\widetilde{g}_{F}$. Again, by (A) this is in contradiction with (C) that $g_{F}$ is not conformally Einstein. This completes the proof.

We emphasize that as a byproduct of this theorem we get explicit formulae, in a coordinate system $\left(t, u, x, y, y^{\prime}, y^{\prime \prime}, z\right)$, for an 8-parameter family of strictly $\mathrm{G}_{2(2)^{-}}$ metrics in dimension seven. These metrics have signature $(4,3)$ and are explicitely given in formula (4.1). In Proposition 4.1 we also give the explicit expressions for a parallel spinor $\psi$ for these metrics and furthermore the explicit expressions for the corresponding closed and coclosed threeform $\omega$ defining the $\mathrm{G}_{2(2)}$-structure on the ambient space $\left(\tilde{M}, \widetilde{g}_{F}\right)$.

\section{Note added in proof}

Recently, C. R. Graham and T. Willse proved in [18] a generalisation of our main result in Theorem 1.1. First they showed that for any $(2,3,5)$-distribution and the associated conformal structure defined as above, the holonomy of the ambient metric is contained in $\mathrm{G}_{2(2)}$. Secondly, they presented an algebraic non-degeneracy condition on the Weyl and the Cotton tensors of this conformal structure under which the holonomy of the ambient metric is equal to $\mathrm{G}_{2(2)}$. In order to prove the second result, they used arguments developed in the present paper in order to rule out a further reduction of the holonomy. Note that their algebraic nondegeneracy condition is far from being necessary for the ambient holonomy to be equal to $\mathrm{G}_{2(2)}$. In particular, the conformal classes in (2) of our Theorem 1.1 do not satisfy this condition. Furthermore, while the present paper was in the submission and editing process, in [27], amongst other results, we proved a tractorial version of statement (B) on page 5 (see also Theorem 2.6). This tractorial version is stronger than statement (B) in the sense that it provides an equivalent characterisation of conformal classes containing a metric admitting a parallel null line bundle $L$ with $\operatorname{Ric}\left(L^{\perp},.\right)=0$ in terms of parallel 2-plane bundles of the tractor bundle. See Remark 2.5 for a related statement. 
ACKNOWLEDGements. We wish to thank Matthias Hammerl for reading the preliminary version of this article and pointing out to us a gap in our reasoning, which we eliminated in the final version. We also acknowledge helpful discussions with Andrzej Trautman, who provided us with a very useful representation of the real Clifford agebra $\mathrm{Cl}(4,3)$, which we use in Section 4. The second author wishes to thank Rod Gover, Jerzy Lewandowski, and the Mathematical Institute of Polish Academy of Sciences for making possible his trip to the Southern Hemisphere, where the final version of this paper was prepared.

\section{The ambient metric of an odd-dimensional conformal structure}

An important tool in conformal geometry is the Fefferman-Graham ambient metric (see [14] and [15] for the following). For a conformal class [ $g]$ in signature $(p, q)$ on an $n=(p+q)$-dimensional manifold $M$ one considers the cone

$$
\mathcal{C}=\left\{\left(g_{p}, p\right) \mid p \in M, g \in[g]\right\}
$$

We denote by $\pi: \mathcal{C} \rightarrow M$ the canonical projection and by $\pi_{*}: T \mathcal{C} \rightarrow T M$ its differential. $\mathcal{C}$ is equipped with an obvious $\mathbb{R}_{+}$-action $\varphi_{t}\left(g_{p}, p\right)=\left(t^{2} g_{p}, p\right)$ and with the tautological tensor $G$ defined by

$$
G_{\left(g_{p}, p\right)}(U, V):=g_{p}\left(\pi_{*}(U), \pi_{*}(V)\right) .
$$

The $\mathbb{R}_{+}$-action extends to $\mathcal{C} \times \mathbb{R}$. Now, the the ambient space $\tilde{M}$ with ambient metric $\widetilde{g}$ is defined by the following properties:

(1) $\tilde{M}$ is an invariant neighbourhood of $\mathcal{C}$ in $\mathcal{C} \times \mathbb{R}$ under the $\mathbb{R}_{+}$action.

(2) $\widetilde{g}$ is a smooth metric of signature $(p+1, q+1)$ on $\tilde{M}$ that is homogeneous of degree two with respect to the $\mathbb{R}_{+}$-action and such that its pullback by $\iota: \mathcal{C} \rightarrow$ $\widetilde{M}$ gives the tautological tensor $G$, i.e. $\iota^{*} \widetilde{g}=G$.

(3) The Ricci tensor $\widetilde{\operatorname{Ric}}$ of $(\widetilde{M}, \widetilde{g})$ is zero.

In the following we are only interested in the case where $M$ is odd-dimensional. In this case, Fefferman and Graham proved the following result.

Theorem $2.1([\mathbf{1 4}, 15]$ and $[24])$. Let $(M,[g])$ be a real analytic manifold $M$ of odd dimension $n>2$ equipped with a conformal structure defined by a real analytic semi-Riemannian metric $g$. Then there exists an ambient space $(\widetilde{M}, \widetilde{g})$ with real analytic Ricci-flat metric $\widetilde{g}$. The ambient space is unique modulo diffeomorphisms that restrict to the identity along $\mathcal{C} \subset \widetilde{M}$ and commute with the $\mathbb{R}_{+}$-action.

By the uniqueness of the ambient metric, its pseudo-Riemannian holonomy is an invariant object of the conformal class: Two different ambient metrics corresponding to two different metrics from the same conformal class are isometric. 
Since the holonomy group is a pseudo-Riemannian invariant, the holonomy group of the ambient metric in odd dimensions is a conformal invariant.

Every metric $g$ in the conformal class $[g]$ defines an embedding

$$
\iota_{g}: M \ni p \mapsto\left(g_{p}, p\right) \in \mathcal{C}
$$

and thus an identification of $\tilde{M}$ with $\mathbb{R}_{+} \times M \times \mathbb{R}$ via

$$
(t, p, \rho) \mapsto\left(t^{2} g_{p}, \rho\right) .
$$

Using this identification and starting with a formal power series

$$
\tilde{g}=2(t \mathrm{~d} \rho+\rho \mathrm{d} t) \mathrm{d} t+t^{2}\left(g+\sum_{k=1}^{\infty} \rho^{k} \mu_{k}\right)
$$

with certain symmetric $(2,0)$ tensors $\mu_{k}$ on $M$, Fefferman and Graham showed that if $n$ is $o d d$, the Ricci-flatness of the ambient metric gives equations for $\mu_{1}, \mu_{2}, \ldots$ that can be solved. However, the $\mu_{k}$ have been determined for small $k$, or for all $k$ but very special conformal classes. For example, in general, one finds that $\mu_{1}=2 \mathrm{P}^{g}$, where $\mathrm{P}$ denotes the Schouten tensor of $g$, and that

$$
\mu_{2}=-B^{g}+\operatorname{tr}\left(\mathrm{P}^{g} \otimes \mathrm{P}^{g}\right)
$$

with $B^{g}$ being the Bach tensor of $g$. Furthermore, for an Einstein metric with $\mathrm{P}^{g}=\Lambda g$ we have that $\mu_{2}=\Lambda^{2} g$ and all other $\mu_{i}=0$, i.e. the power series in the ambient metric $\widetilde{g}_{E}$ truncates at $k=2$. Further calculations of the ambient metric have been carried out for conformal classes that are related to Einstein spaces [16]. However, if the metric $g$ is not conformally Einstein, then, except for a few examples [16,28,32], no explicit formulae for $\mu_{k}, k>3$ are known.

For further convenience we change the coordinate $\rho$ on $\widetilde{M}$ to $u:=-\rho$ t, i.e. $\mathrm{d} u=-t \mathrm{~d} \rho-\rho \mathrm{d} t$. Then the ambient metric takes the form

$$
\tilde{g}=-2 \mathrm{~d} u \mathrm{~d} t+t^{2} g-2 u t \mathrm{P}^{g}+u^{2}\left(\mu_{2}-\frac{u}{t} \mu_{3}+\left(\frac{u}{t}\right)^{2} \mu_{4}-\ldots\right) .
$$

In particular, for a Ricci-flat metric, the ambient metric is given as a special Brinkmann wave,

$$
\widetilde{g}=-2 \mathrm{~d} u \mathrm{~d} t+t^{2} g
$$

admitting a parallel null vector field, whereas for an Einstein metric with $\mathrm{P}=\Lambda g$ the ambient metric becomes

$$
\tilde{g}=-2 \mathrm{~d} u \mathrm{~d} t+\left(t^{2}-2 \Lambda u t+\Lambda^{2} u^{2}\right) g
$$

This metric splits into a line and a cone. This becomes evident in new coordinates $r=t-\Lambda u$ and $s=t+\Lambda u$ in which we have

$$
\tilde{g}=\frac{1}{2 \Lambda}\left(\mathrm{d} r^{2}-\mathrm{d} s^{2}\right)+r^{2} g .
$$


Now, let $\widetilde{g}$ be the ambient metric for an arbitrary conformal class [ $g$ ] on an odddimensional manifold. We calculate the Levi-Civita connection $\widetilde{\nabla}$ of $\widetilde{g}$ along $\mathcal{C}=$ $\{u=0\}$ and obtain that the only non-vanishing terms are

$$
\left.\begin{array}{rl}
\widetilde{\nabla}_{X} \frac{\partial}{\partial u} & =-\frac{1}{t} \mathrm{P}^{g}(X)^{*} \\
\widetilde{\nabla}_{X} Y & =\nabla_{X} Y+t\left(g(X, Y) \frac{\partial}{\partial u}-\mathrm{P}^{g}(X, Y) \frac{\partial}{\partial t}\right) \\
\widetilde{\nabla}_{X} \frac{\partial}{\partial t} & =\frac{1}{t} X
\end{array}\right\},
$$

for $X, Y \in \Gamma(T M)$ and $\nabla$ being the Levi-Civita connection of $g$.

In the following we need the transformation of the Schouten tensor under a conformal rescaling. Recall that if $\hat{g}=\mathrm{e}^{2 \Upsilon} g$, with $\Upsilon \in C^{\infty}(M)$, is a conformally changed metric, then the Schouten tensor $\hat{P}$ of $\hat{g}$ satisfies

$$
\hat{\mathrm{P}}=\mathrm{P}-\operatorname{Hess}^{g}(\Upsilon)+\mathrm{d}^{2}-\frac{1}{2}\left\|\operatorname{grad}^{g}(\Upsilon)\right\|_{g}^{2} g
$$

where Hess $(\Upsilon)=g\left(\nabla \operatorname{grad}^{g}(\Upsilon),.\right)$ denotes the Hessian of $\Upsilon$. For brevity we will also write this relation as

$$
\hat{\mathrm{P}}_{a b}=\mathrm{P}_{a b}-\nabla_{a} \Upsilon_{b}+\Upsilon_{a} \Upsilon_{b}-\frac{1}{2} \Upsilon_{c} \Upsilon^{c} g_{a b}
$$

Hence, $\hat{g}$ is an Einstein metric if and only if this quantity is a multiple of the metric $g$. More explicitly, it holds that $\hat{\mathrm{P}}_{a b}=\Lambda \hat{g}_{a b}$, where $\Lambda$ is a constant, if and only if

$$
\mathrm{P}_{a b}-\nabla_{a} \Upsilon_{b}+\Upsilon_{a} \Upsilon_{b}=\left(\frac{1}{2} \Upsilon_{c} \Upsilon^{c}+\Lambda \mathrm{e}^{2 \Upsilon}\right) g_{a b} .
$$

By substituting $\Upsilon=-\log (\sigma)$ for a non vanishing function $\sigma$, we obtain that the metric $\hat{g}=\sigma^{-2} g=\mathrm{e}^{2 \Upsilon} g$ is Einstein if and only if there is an non vanishing function $\sigma$ such that the symmetric tensor $\operatorname{Hess}(\sigma)+\sigma \mathrm{P}$ is a multiple of $g$. Explicitly, we have

$$
\nabla_{a} \sigma_{b}+\sigma \mathrm{P}_{a b}=\sigma^{-1}\left(\Lambda+\frac{1}{2} \sigma_{c} \sigma^{c}\right) g_{a b} .
$$

Now we give a characterization of locally conformally Einstein metrics in terms of their ambient metric.

Theorem 2.2. Let $M$ be an odd dimensional manifold equippped with a real analytic conformal class $[\mathrm{g}]$. If the ambient space $(\widetilde{M}, \widetilde{g})$ admits a line bundle $\mathcal{L}$ that is parallel with respect to the Levi-Civita connection of $\widetilde{g}$, then on the connected components of an open dense subset $M_{0}$ in $M$, every metric in the conformal class $[g]$ is locally conformal to an Einstein metric $g_{E}$.

Furthermore, if the ambient metric on $\mathcal{L}$ is positive/negative/zero, then the constant $\Lambda$ in $\mathrm{P}=\Lambda g_{E}$ is negative/positive/zero. 
Proof. We start the proof with a lemma.

Lemma 2.3. There is no open set $U$ in $\mathcal{C}$ such that $\left.\left.\mathcal{L}\right|_{U} \subset T \mathcal{C}\right|_{U}$.

Proof. Assume that we have an open set $U \subset \mathcal{C}$ such that $\left.\left.\mathcal{L}\right|_{U} \subset T \mathcal{C}\right|_{U}$. Since $\mathcal{L}$ is parallel, by making $U$ smaller, such that it becomes simply connected, we can assume that there is a section $L \in \Gamma\left(\left.\mathcal{L}\right|_{U}\right)$. We fix a metric $g \in[g]$ to obtain $(t, x)$-coordinates on $U$ and write

$$
L=\alpha \partial_{t}+K,
$$

with $K$ tangential to $M$. Since $\mathcal{L}$ is parallel, formula (2.5) implies

$$
0 \equiv \widetilde{g}\left(\widetilde{\nabla}_{X} L, \partial_{t}\right)=-\widetilde{g}\left(L, \widetilde{\nabla}_{X} \partial_{t}\right)=\frac{1}{t} \widetilde{g}(X, K)=\operatorname{tg}(X, K),
$$

for all $X \in T M$. This implies $K \equiv 0$ on $U$. Hence $L=\alpha \partial_{t}$, but this contradicts $\widetilde{\nabla}_{X} \partial_{t}=\frac{1}{t} X$ for all $X \in T M$.

This lemma implies that there is an open dense set $\mathcal{C}_{0}$ in $\mathcal{C}$ such that $\left.\mathcal{L}\right|_{\mathcal{C}_{0}} \not \subset$ $\left.T \mathcal{C}\right|_{\mathcal{C}_{0}}$. For every point in $M_{0}:=\pi\left(\mathcal{C}_{0}\right)$ we have to verify the existence of a neighbourhood on which a metric in $[g]$ can be rescaled to an Einstein metric. The following lemma will be useful.

Lemma 2.4. On every simply connected open subset $U$ of the open and dense subset $\mathcal{C}_{0}$ in $\mathcal{C}$ there is a section $L \in \Gamma\left(\left.\mathcal{L}\right|_{U}\right)$ such that $\left.\widetilde{\nabla}_{Y} L\right|_{U}=0$ for all $Y \in T \mathcal{C}$.

Proof. For every simply connected open set $U$ in $\mathcal{C}_{0}$ we find a section of $\mathcal{L}$ which, by fixing $g \in[g]$ and by the previous lemma, is of the form

$$
L=a \partial_{t}+K+\partial_{u}
$$

with $K$ tangential to $M$. Since $\mathcal{L}$ is parallel, there is a 1-form $\Theta$ over $U$ such that $\widetilde{\nabla} L=\Theta \otimes L$. We will show that $\Theta$ is closed, which implies that $L$ can be rescaled to a parallel vector field. The following calculations are over $U$. We get

$$
\Theta\left(\partial_{t}\right)=-\widetilde{g}\left(\widetilde{\nabla}_{\partial_{t}} L, \partial_{t}\right) \equiv 0,
$$

which implies that

$$
K=\frac{1}{t} K_{0}
$$

for a $K_{0} \in \Gamma(T M)$. Indeed, for every $X \in T M$ it is

$$
\widetilde{g}\left(\widetilde{\nabla}_{\partial_{t}} L, X\right)=\widetilde{g}\left(\widetilde{\nabla}_{\partial_{t}} K, X\right)=\widetilde{g}\left(\left[\partial_{t}, K\right], X\right)+\frac{1}{t} \widetilde{g}(K, X) .
$$

Now, as $\widetilde{g}\left(\widetilde{\nabla}_{\partial_{t}} L, X\right)=\Theta\left(\partial_{t}\right) \widetilde{g}(K, X)=0$, this implies that $K$ satsifies the equation

$$
\left[\partial_{t}, K\right]=-\frac{1}{t} K,
$$


which yields $K=\frac{1}{t} K_{0}$ with $K_{0} \in \Gamma(T M)$. Furthermore it is

$$
\Theta(X)=-\widetilde{g}\left(\widetilde{\nabla}_{X} L, \partial_{t}\right)=\widetilde{g}\left(L, \widetilde{\nabla}_{X} \partial_{t}\right)=\frac{1}{t} \widetilde{g}(K, X)=g\left(K_{0}, X\right)
$$

for $X \in T M$. Hence, in order to show that $\Theta$ is closed we only have to check $\mathrm{d} \Theta(X, Y)=0$ for $X, Y \in T M$. On the one hand we get that

$$
\widetilde{g}\left(\widetilde{\nabla}_{X} L, Y\right)=t\left(\operatorname{ag}(X, Y)+\operatorname{tg}\left(\nabla_{X} K_{0}, Y\right)-P^{g}(X, Y)\right),
$$

and on the other that

$$
\widetilde{g}\left(\widetilde{\nabla}_{X} L, Y\right)=t \Theta(X) g(L, Y)=t \Theta^{2}(X, Y),
$$

which shows that $g\left(\nabla_{X} K_{0}, Y\right)$ is symmetric in $X, Y \in T M$. But this implies that $\Theta=g\left(K_{0},.\right)$ is closed. Hence, on simply connected open sets $U \subset \mathcal{C}_{0}$ we get that $\Theta=\mathrm{d} f$ which implies that $\mathrm{e}^{-f} \cdot L$ is a parallel vector field on $U$.

Now we conclude the proof of the theorem by fixing a metric $g$ in $[g]$ and showing that it can be rescaled to an Einstein metric $g_{E}$ on simply connected open sets in $M$. By the lemmas, on simply connected open sets in $\mathcal{C}_{0}$ we get a parallel vector field

$$
L=\alpha \partial_{t}+K+\sigma \partial_{u} \in \Gamma\left(\left.\mathcal{L}\right|_{U}\right),
$$

with $\sigma \neq 0$ and $K$ tangential to $M$. Again, $\widetilde{\nabla}_{\partial_{t}} L=0$ implies that $K=\frac{1}{t} K_{0}$ with $\widetilde{V}_{0} \in \Gamma(T M)$, but also that $\mathrm{d} \sigma\left(\partial_{t}\right)=\mathrm{d} \alpha\left(\partial_{t}\right) \equiv 0$. For $X \in T M$, the equation $\widetilde{\nabla}_{X} L=0$ implies

$$
\begin{aligned}
\mathrm{d} \sigma(X)+g\left(X, K_{0}\right) & =0 \\
\mathrm{~d} \alpha(X)-P\left(X, K_{0}\right) & =0 \\
g\left(\nabla_{X} K_{0}, Y\right)-\sigma P^{g}(X, Y)+\alpha g(X, Y) & =0 .
\end{aligned}
$$

The first equation shows that $K_{0}=-\operatorname{grad}^{g}(\sigma)$. Then the last equation shows

$$
\operatorname{Hess}(\sigma)+\sigma P^{g}=\alpha g .
$$

But this is equivalent to $\sigma^{-2} g$ being a local Einstein metric. Note that (2.7) implies that

$$
\sigma \alpha=\left(\hat{\Lambda}+\frac{1}{2} g\left(K_{0}, K_{0}\right)\right)
$$

with the Einstein constant $\hat{\Lambda}$ of $\sigma^{-2} g$. But this implies that

$$
\widetilde{g}(L, L)=-2 \alpha \sigma+g\left(K_{0}, K_{0}\right)=-2 \hat{\Lambda},
$$

which shows the relation between the Einstein constant and the line bundle being zero, positive, or negative. 
Remark 2.5. Using the formulae in (2.5), one can show that the connected component of the normal conformal Cartan connection for the conformal structure $[g]$ is contained in the holonomy of the ambient metric $\tilde{g}$. Furthermore, when the conformal class contains an Einstein metric, the truncation of the ambient metric in this case yields the equality of both holonomy groups (see [26] and [30]). This can be used to prove an analogue of Theorem 2.2 in terms of the normal conformal Cartan connection. This analogue holds in any dimension and gives an equivalence between the existence of a parallel line $\mathcal{L}$ in $(\widetilde{M}, \widetilde{g})$ and an Einstein metric $g_{E}$ in $[g]$. As we will use here only one direction and only in odd dimensions, for the purpose of being self contained, we did prove Theorem 2.2 without referring to the normal conformal Cartan connection and without using tractor calculus. For further results relating the ambient metric and tractor calculus, see $[3,9,26]$.

Now we will describe the case where the Levi-Civita connection of the ambient metric admits an invariant null 2-plane ${ }^{1}$. We will deal with a bit more general situation than needed for our pourposes, i.e. with the case when the 2-plane is totally null in arbitrary signature. The following theorem is a generalisation to arbitrary signature of the corresponding result from the Lorentzian domain, which was proved in [26].

Theorem 2.6. Let $(M,[g])$ be a pseudo-Riemannian real analytic conformal manifold of odd dimension $n>2$. If the holonomy group of the ambient metric admits an invariant totally null 2-plane, then every metric in the conformal class $[g]$ is locally conformally equivalent to a metric $g$ with the following two properties:

There is a null line $L \subset T M$ that is parallel for $\nabla^{g}$, and

$$
\operatorname{Ric}^{g}(Y)=0 \text { for all } Y \in L^{\perp} \text {. }
$$

Remark 2.7. Note that property (2.12) is equivalent to the property that the image of $\mathrm{Ric}^{g}: T M \rightarrow T M$ is contained in $L$. In Lorentzian signature, this is equivalent to the image of $\mathrm{Ric}^{g}$ being totally null. In higher signature, it is stronger.

Note also that such metrics have vanishing scalar curvature, and thus Ric is a constant multiple of the Schouten tensor. It also holds that Ric satsifies property (2.12) if and only if the Schouten satisfies property (2.12).

Proof. The proof is based on the following Lemma.

Lemma 2.8. Let $(\tilde{M}=\mathcal{C} \underset{\sim}{\mathbb{R}}, \widetilde{g})$ be the ambient space and let $\mathcal{H}$ be a bundle of parallel null 2-planes on $(\widetilde{M}, \widetilde{g})$. Assume that there is a bundle of null lines L over $M$ and a metric $g \in[g]$ defining the embedding $\iota_{g}: M \rightarrow \mathcal{C}$ such that

$$
\left.\mathcal{H}\right|_{l_{g}(M)}=\left(\iota_{g}\right)_{*}(L) \oplus \mathbb{R} \partial_{u} .
$$

Then $L$ is parallel with respect to $\nabla^{g}$ and $\left.Y\right\lrcorner \operatorname{Ric}^{g}=0$ for all $Y \in L^{\perp}$.

1 The case where the 2-plane is non-degenerate implies that the conformal class contains a product of Einstein metrics with related Einstein constants (see [2] for Riemannian conformal classes and the unpublished parts in [29] for arbitrary signature). 
Proof. Let $g \in[g]$ be the metric given in the assumptions and let $\widetilde{M}=\mathbb{R}_{+} \times$ $\iota_{g}(M) \times \mathbb{R} \ni(t, p, u)$. Furthermore, let $V=a \partial_{u}+K \in \Gamma\left(\left.\mathcal{H}\right|_{\iota_{g}(M)}\right)$ with $K \in \Gamma(L)$ a null vector. Then, along $\iota_{g}(M) \subset \mathcal{C}$, by formulae (2.5), we get

$$
0=\widetilde{g}\left(\widetilde{\nabla}_{X} V, \partial_{u}\right)=-\widetilde{g}\left(\widetilde{\nabla}_{X} \partial_{u}, K\right)=t P^{g}(X, K),
$$

for all $X \in T M$. This shows that $L\lrcorner P^{g}=0$. In particular, the image of $\mathrm{Ric}^{g}$ and hence the image of $\mathrm{P}^{g}$ lies in $L$. Furthermore,

$$
\tilde{\nabla}_{X} V=X(a) \partial_{u}+\frac{a}{t} \mathrm{P}^{g}(X)+\nabla_{X}^{g} K+\operatorname{tg}(X, K) \partial_{u},
$$

for all $X \in T M$. Since $\left.\mathcal{H}\right|_{l g}(M)=L \oplus \mathbb{R} \partial_{u}$ is parallel, and since $\mathrm{P}^{g}(X) \in L$, this implies that $L$ is parallel with respect to $\nabla^{g}$.

We will now show that the existence of a parallel totally null 2-plane distribution on $(\widetilde{M}, \widetilde{g})$ implies the existence of a metric in the conformal class and a null line bundle on $M$ satisfying the assumptions of Lemma 2.8 .

Let $\mathcal{H}$ be a totally null 2-plane bundle that is parallel for the ambient metric. With $\mathcal{H}$ also $\mathcal{H}^{\perp}$ is parallel, but of rank $n$. This implies

$$
\left.\mathcal{H}\right|_{\mathcal{C}} \not \subset T \mathcal{C} \text { and }\left.\mathcal{H}^{\perp}\right|_{\mathcal{C}} \not \subset T \mathcal{C} \text {. }
$$

In order to prove this, fix a metric $g \in[g]$. Since $\mathcal{H}$ has rank $2,\left.\mathcal{H}\right|_{\mathcal{C}} \subset T \mathcal{C}$ would imply that there is a section $K \in \Gamma\left(\left.\mathcal{H}\right|_{\mathcal{C}}\right)$ that is tangential to $\iota_{g}(M)$. $\mathcal{H}$ being parallel then gives

$$
0=\widetilde{g}\left(\widetilde{\nabla}_{X} K, \partial_{t}\right)=-\widetilde{g}\left(\widetilde{\nabla}_{X} \partial_{t}, K\right)=-\operatorname{tg}(X, K),
$$

for all $X$ in $T M$, which contradicts the non-degeneracy of the metric. Then, since $\mathcal{H}$ is totally null, and thus $\mathcal{H} \subset \mathcal{H}^{\perp}$, property (2.13) follows. For reasons of dimensions, this implies that

$$
\mathcal{L}:=\left.\mathcal{H}\right|_{\mathcal{C}} \cap T \mathcal{C} \text { is a bundle of null lines over } \mathcal{C} .
$$

We will now prove some properties of $\mathcal{L}$ that will lead to the proof of the theorem.

Lemma 2.9. Let $\mathcal{X}:=\mathcal{L}^{\perp} \cap T \mathcal{C}$ and $L \in \Gamma(\mathcal{L})$. Then

$$
\widetilde{g}\left(\widetilde{\nabla}_{U} L, V\right)=0 \text { for all } U, V \in \mathcal{X} .
$$

Proof. We show that for $U \in \mathcal{X}$ the vector field $\widetilde{\nabla}_{U} L$ is not only contained in $\mathcal{H}$, by $\mathcal{H}$ being parallel, but also in the space tangential to the cone, and hence in $\mathcal{L}$. To this end we fix a metric $g \in[g]$ yielding $\mathcal{C}=\mathbb{R}_{+} \times M$ and $T \mathcal{C}=\partial_{t}^{\perp}$. Hence, for $L=a \partial_{t}+K \in \mathcal{L}$ and $U=b \partial_{t}+X \in \mathcal{X}$, with $K$ and $X$ tangential to $M$ and orthogonal to each other, $\widetilde{g}\left(\widetilde{\nabla}_{U} L, \partial_{t}\right)=-\widetilde{g}\left(L, \widetilde{\nabla}_{U} \partial_{t}\right)=-\widetilde{g}\left(L, \widetilde{\nabla}_{X} \partial_{t}\right)=-\frac{1}{t} \widetilde{g}(L, X)=-\frac{1}{t} \widetilde{g}(X, K)=0$, since $g(K, X)=0$ and $\partial_{t}^{\perp}=T \mathcal{C}$. This shows that $\widetilde{\nabla}_{U} L \in \mathcal{H} \cap T \mathcal{C}$ implying the relation (2.15). 
Lemma 2.10. $\mathcal{X}=\mathcal{L}^{\perp} \cap T \mathcal{C}$ is an integrable distribution on $\mathcal{C}$.

Proof. We fix a metric $g \in[g]$ and obtain $M \hookrightarrow \mathcal{C}$. First note that $\mathcal{H}^{\perp} \subset \mathcal{L}^{\perp}$ and that $\partial_{t} \in \mathcal{L}^{\perp}$. Furthermore $\mathcal{H}^{\perp} \cap \partial_{t}=\{0\}$. Indeed, since $\mathcal{H} \not \subset T \mathcal{C}$, there is an element in $\mathcal{H}$ of the form $\partial_{u}+X+a \partial_{t}$, which implies that $\partial_{t}$ is not orthogonal to $\mathcal{H}$. Furthermore, since $\mathcal{H}^{\perp} \subset \mathcal{L}^{\perp}$, by relation (2.13), the dimension of $\mathcal{L}^{\perp} \cap \mathcal{T C}$ is $n$. Now, let $\partial_{t}, Y_{1}, \ldots, Y_{n-1}$ with $Y_{i} \in \Gamma\left(\mathcal{H}^{\perp} \cap T \mathcal{C}\right)$ be basis for $\mathcal{L}^{\perp} \cap T \mathcal{C}$ of mutually orthogonal vector fields. Then, since $\mathcal{H}^{\perp}$ is parallel we have $\widetilde{\nabla}_{\partial_{t}} Y_{i} \in \Gamma\left(\mathcal{H}^{\perp}\right)$. Hence,

$$
\left[\partial_{t}, Y_{i}\right]=\widetilde{\nabla}_{\partial_{t}} Y_{i}-\widetilde{\nabla}_{Y_{i}} \partial_{t}=\widetilde{\nabla}_{\partial_{t}} Y_{i}-\frac{1}{t} Y_{i} \in \mathcal{H}^{\perp} \cap T \mathcal{C} .
$$

On the other hand, again since $\mathcal{H}^{\perp}$ is parallel, we get $\widetilde{\nabla}_{Y_{i}} Y_{j} \in \Gamma\left(\mathcal{H}^{\perp}\right)$ and thus $\left[Y_{i}, Y_{j}\right] \in \Gamma\left(\mathcal{H}^{\perp}\right)$. Furthermore,

$$
\widetilde{g}\left(\left[Y_{i}, Y_{j}\right], \partial_{t}\right)=\widetilde{g}\left(\widetilde{\nabla}_{Y_{i}} Y_{j}-\widetilde{\nabla}_{Y_{j}} Y_{i}, \partial_{t}\right)=-\widetilde{g}\left(Y_{j}, \widetilde{\nabla}_{Y_{i}} \partial_{t}\right)+\widetilde{g}\left(Y_{i}, \widetilde{\nabla}_{Y_{j}} \partial_{t}\right)=0,
$$

because of (2.5). This shows that also $\left[Y_{i}, Y_{j}\right] \in \Gamma(\mathcal{H} \cap T \mathcal{C})$. Hence, $\mathcal{H}^{\perp} \cap T \mathcal{C}$ but also $\mathcal{L}^{\perp} \cap T \mathcal{C}$ are integrable.

Lemma 2.11. Let $\pi: \mathcal{C} \rightarrow M$ and $\pi_{*}: T \mathcal{C} \rightarrow T M$ be the canonical projection and its differential. Then

$$
L:=\pi_{*}(\mathcal{L})
$$

is a distribution of null lines on $M$ and $L^{\perp}=\pi_{*}(\mathcal{X})$. Both distributions are integrable on $M$.

Proof. We fix $g \in[g]$ to verify that $\pi_{*}(\mathcal{L}) \neq 0$. Assume that $\mathcal{L}=\mathbb{R} \partial_{t}$. $\mathcal{H}$ being parallel then implies that

$$
\widetilde{\nabla}_{X} \partial_{t}=\frac{1}{t} X \in \mathcal{H} \text { for all } X \in T M .
$$

Since $n>2$, this contradicts to $\mathcal{H}$ being a 2-plane bundle. Hence, $\pi_{*}(\mathcal{L})$ is a null line bundle on $M$. Since $T \mathcal{C}=\partial_{t}^{\perp}$ this implies that $L^{\perp}=\mathcal{L}^{\perp} \cap T \mathcal{C}=\mathcal{X}$. Since $\mathcal{L}$ and $\mathcal{X}$ are integrable on $\mathcal{C}, L$ and $L^{\perp}$ are integrable on $M$.

Lemma 2.12. For any null vector $K \in \Gamma(L)$ we define the second fundamental form of $L^{\perp}$ by

$$
\Pi^{K}(X, Y)=g\left(\widetilde{\nabla}_{X} K, Y\right) \text { for } X, Y \in L^{\perp} .
$$

Then $\Pi^{K}$ is symmetric and tensorial in $K$. Furthermore, locally there is a metric in the conformal class such that $\Pi^{K}(X, Y)$ has no trace. 
Proof. First we notice that $L \subset L^{\perp}$ implies that $\Pi^{K}$ is tensorial in $K$ : For $f K$ with a smooth function $f$ we get

$$
\Pi^{f k}(X, Y)=X(f) \widetilde{g}(K, X)+f \widetilde{g}\left(\widetilde{\nabla}_{X} K, Y\right)=f \Pi^{K}(X, Y) .
$$

The integrability of $L^{\perp}$ implies that $\Pi^{K}$ is symmetric. Now we define the trace of $\Pi^{K}$ as

$$
H^{K}:=\sum_{i=1}^{n-2} \varepsilon_{i} \Pi^{K}\left(E_{i}, E_{i}\right) \in C^{\infty}(M)
$$

where $E_{1}, \ldots, E_{n-2}$ linearly independent in $L^{\perp}$ with $g\left(E_{i}, E_{j}\right)=\varepsilon_{i} \delta_{i j}$. Since $K\lrcorner \Pi^{K}=0$, this is independent of the chosen $E_{i}$ 's. Now we claim that there is a metric $\hat{g}=\mathrm{e}^{2 \Upsilon} g \in[g]$ in the conformal class such that the corresponding function $\hat{H}^{K}$ is zero. To this end we notice that the transformation formula for $\hat{\Pi}^{K}$ is given by

$$
\hat{\Pi}^{K}(Y, V)=\hat{g}\left(\hat{\nabla}_{Y} K, V\right)=\mathrm{e}^{2 \Upsilon}(\Pi(Y, V)+\mathrm{d} \Upsilon(K) g(V, Y)),
$$

for $Y, V \in L^{\perp}$. Hence,

$$
\hat{H}^{K}=\mathrm{e}^{2 \Upsilon}\left(H^{K}+(n-2) \mathrm{d} \Upsilon(K)\right) .
$$

Now the differential equation

$$
\mathrm{d} \Upsilon(K)=\frac{H^{K}}{n-2}
$$

is an ODE along the flows of $K$ and as such locally always has a solution. This ensures that we can chose $\hat{g}$ such that $\hat{H}^{K} \equiv 0$.

Finally, to conclude the proof, we fix this metric $g \in[g]$ for which $H^{K} \equiv 0$. Now let $L=a \partial_{t}+K \in \Gamma(\mathcal{L})$ be arbitrary. Then equation $(2.15)$ reads as

$$
0=\widetilde{g}\left(\widetilde{\nabla}_{X} L, Y\right)=\operatorname{atg}(X, Y)+t^{2} \Pi^{K}(X, Y),
$$

for all $X, Y \in L^{\perp}$. Taking the trace shows that $a \equiv 0$ on $M$. Hence, $\mathcal{L}=\iota_{g}(L)$, and thus $\mathcal{H}=\iota_{g}(L) \oplus \mathbb{R} \partial_{u}$. This means that the metric $g \in[g]$ and the null line bundle $L$ on $M$ satisfy the assumptions of Lemma 2.8. This concludes the proof of the theorem.

\section{3. $G_{2(2)}$-conformal structures with truncated ambient metric}

As it was mentioned in the Introduction, in [31] a conformal structure $\left[g_{F}\right]$ in signature $(3,2)$ was introduced that originated from a first order ODE for two functions 
$y, z$ of one variable $x$. We will now describe this construction briefly. Every solution to the first order ODE

$$
z^{\prime}=F\left(x, y, y^{\prime}, y^{\prime \prime}, z\right) \quad \text { with } \quad F_{y^{\prime \prime} y^{\prime \prime}} \neq 0,
$$

is a curve in the five-dimensional manifold $M$ parametrised by $\left(x, y, z, p=y^{\prime}, q=\right.$ $\left.y^{\prime \prime}\right)$, on which the one-forms

$$
\omega^{1}=\mathrm{d} z-F(x, y, p, q, z) \mathrm{d} x, \omega^{2}=\mathrm{d} y-p \mathrm{~d} x, \omega^{3}=\mathrm{d} p-q \mathrm{~d} x
$$

vanish. Two triples of such 1 -forms on $\mathbb{R}^{5},\left(\omega^{1}, \omega^{2}, \omega^{3}\right)$ and $\left(\hat{\omega}^{1}, \hat{\omega}^{2}, \hat{\omega}^{3}\right)$, are considered to be equivalent, if there is a local diffeomorphism $\Phi$ of $\mathbb{R}^{5}$ and a $\operatorname{GL}(3, \mathbb{R})$ valued function $A=\left(a_{j}^{i}\right)$ on the domain of $\Phi$ such that $\Phi^{*} \hat{\omega}^{i}=\sum_{j=1}^{n} a_{j}^{i} \omega^{j}$. Cartan showed that an equivalence class of a triple of one-forms given by (3.1) with $F_{q q} \neq 0$ corresponds to a Cartan connection $\omega$ on a 14-dimensional principle fibre bundle $\mathcal{P}$ over the five-manifold parametrised by $(x, y, z, p, q)$. This Cartan connection has values in the non-compact exceptional Lie algebra $\mathfrak{g}_{2(2)}$, and $\mathcal{P}$ is the bundle with structure group given by the 9 -dimensional parabolic $P:=\mathrm{G}_{2(2)} \cap B$, where $B$ is the isotropy group in $\mathrm{SO}(4,3)$ of a null line. The conformal structure on the 5-manifold is now constructed as follows: Write the Cartan connection $\omega$ as $\omega=(\theta, \Omega)$, where $\Omega$ has values in the Lie algebra $\mathfrak{p}$ of $P$ and $\theta$ in the five-dimensional complement of $\mathfrak{p}$ in $\mathfrak{g}_{2(2)}$. Write $\theta=\left(\theta_{1}, \ldots, \theta_{5}\right)$ and $\Omega=\left(\Omega_{1}, \ldots, \Omega_{9}\right)$ and let $X_{1}, \ldots, X_{5}$ and $Y_{1}, \ldots, Y_{9}$ be the vector fields on $\mathcal{P}$ dual to $\theta_{i}$ and $\Omega_{\mu}$, respectively. The $Y_{\mu}$ are tangential to the fibres of $\mathcal{P} \rightarrow M$. Defining the bilinear form

$$
G=2 \theta^{1} \theta^{5}-2 \theta^{2} \theta^{4}+\left(\theta^{3}\right)^{2}
$$

on $\mathcal{P}$ we note that along the fibres $G$ is degenerate and merely scales, i.e.

$$
\mathcal{L}_{Y_{\mu}} G=\lambda_{\mu} G
$$

for some functions $\lambda_{\mu}$. Hence, $G$ projects to a conformal class of metrics $\left[g_{F}\right]$ of signature $(+++--)$ on $M$. This means that the normal conformal Cartan connection for $\left[g_{F}\right]$ reduces (in the Cartan sense) to $\mathrm{G}_{2(2)}$. Hence, the conformal holonomy of $\left[g_{F}\right]$ is contained in this group. Of course, this inclusion might be proper.

Remark 3.1. The conformal structure given by $F$ is an example of a conformal Cartan reduction (see for example [1]). The normal conformal Cartan connection of $\left[g_{F}\right]$ reduces to a Cartan connection with values in the Lie algebra $\mathfrak{g}_{2(2)} \subset \mathfrak{s o}(4,3)$. In this way it defines a parabolic geometry of type $\left(P, \mathfrak{g}_{2(2)}\right)$, where $P$ is the parabolic subgroup given by the stabiliser in $\mathrm{G}_{2(2)}$ of a null line. This situation is exceptional in the sense that a reduction of a Cartan connection to a semisimple subalgebra $\mathfrak{g} \subsetneq \mathfrak{s o}(p+1, q+1)$ whose intersection with the stabiliser of a null line is parabolic imposes very strong algebraic restrictions on $\mathfrak{g}$ and the parabolic subalgebra, as recently shown in [13]. For conformal geometry, only two cases arise: the one of $\mathfrak{g}_{2(2)}$, which, by the result in [19], is given by the above construction, and the one of $\mathfrak{s o}(4,3) \subset \mathfrak{s o}(4,4)$ described in [7]. 
Then, in [32], the following remarkable feature of $\left[g_{F}\right]$ was noticed.

Proposition 3.2. There exist functions $F$ such that the ambient metric of a $g_{F} \in$ $\left[g_{F}\right]$ truncates after terms of second order, i.e.

$$
\tilde{g}_{F}=-2 \mathrm{~d} t \mathrm{~d} u+t^{2} g_{F}-2 u t \mathrm{P}+u^{2} \beta,
$$

with $\mathbf{P}$ the Schouten tensor of $g_{F}$ and $\beta=\mu_{2}$ defined as in equation (1.2).

Examples of such $F$ 's given in [32] include $F=F(q)$ and $F=q^{2}+$ $\sum_{i=0}^{6} a_{i} p^{i}+b z$. The proof is based on the form and the uniqueness of the ambient metric in odd dimensions proved in $[14,15]$ and the observation, that the metric (3.2) is Ricci-flat.

This concise form of the ambient metric makes it possible to study the relation between the conformal holonomy and the holonomy of the ambient metric. This is done by distinguishing two situations: the first, when the conformal class contains an Einstein metric, and the second, when it does not contain an Einstein metric. Also in [32] several examples of such conformal structures depending on the function $F$ in (3.1) with $F_{q q} \neq 0$ were considered. On the one hand it was shown that for $F=F(q)$ the conformal class given by $F$ contains a Ricci flat metric. We have seen that for a conformal class that contains a Ricci flat metric, the ambient metric is a special Brinkmann metric, $\widetilde{g}=-2 \mathrm{~d} u \mathrm{~d} t+t^{2} g$, and that the holonomy of the ambient metric is the same as the holonomy of the conformal Cartan connection. Based on the result in [32] we obtain:

Proposition 3.3. Let $\left[g_{F}\right]$ be a conformal class where $F=F(q)$ with $F_{q q} \neq 0$. Then $\left[g_{F}\right]$ contains a Ricci flat metric $g_{0}$, the ambient metric for $\left[g_{F}\right]$ is

$$
\tilde{g}_{F}=-2 \mathrm{~d} u \mathrm{~d} t+t^{2} g_{0}
$$

as in equation (2.3), the holonomy of the ambient metric is equal to the conformal holonomy and contained in the eight-dimensional stabiliser in $\mathrm{G}_{2(2)}$ of a null vector.

This shows that the ambient metric of conformal classes $g_{F(q)}$ are $\mathrm{G}_{2(2)}$-metrics that admit a parallel null vector field, and thus can be considered as $\mathrm{G}_{2(2)}$-Brinkmann waves.

Furthermore, in [32] a conformal structure $\left[g_{F}\right]$ in signature $(3,2)$ was introduced that still has an ambient metric in the truncated form (3.2) but does not contain an Einstein metric. This is defined by

$$
F=q^{2}+\sum_{i=0}^{6} a_{i} p^{i}+b z,
$$

where the conformal class is given by the metric

$$
g_{F}=2 \theta^{1} \theta^{5}-2 \theta^{2} \theta^{4}+\left(\theta^{3}\right)^{2},
$$


where the co-frames $\theta^{i}$ are given by

$$
\theta^{i}=\mathrm{e}^{-\frac{2 b}{3} x} \hat{\theta}^{i}
$$

with the $\hat{\theta}$ 's defined in on page 3 . For further convenience we define

$$
\begin{aligned}
& A_{3}=\frac{9}{20 \cdot 2^{2 / 3}}\left(a_{3}+4 a_{4} p+10 a_{5} p^{2}+20 a_{6} p^{3}\right) \\
& A_{4}=\frac{9}{10}\left(a_{4}+5 a_{5} p+15 a_{6} p^{2}\right), \\
& A_{5}=\frac{27}{4 \cdot 2^{1 / 3}}\left(a_{5}+6 a_{6} p\right), \\
& A_{6}=\frac{243}{2 \cdot 2^{2 / 3}} a_{6} .
\end{aligned}
$$

Note that we use here a different metric in the conformal class $\left[g_{F}\right]$ than in [32]. We have rescaled the metric in [32] by $\mathrm{e}^{-\frac{4 b}{3} x}$ which will give Cotton flat metrics for some $F$ 's. When we write in the following "not conformal" we mean "nowhere locally conformal". Correspondingly, "conformal" for us always means "locally conformal".

Proposition 3.4. If at least one of $a_{4}, a_{5}$, or $a_{6}$ is not zero, then the conformal class $\left[g_{F}\right]$ corresponding to $F=q^{2}+\sum_{i=0}^{6} a_{i} p^{i}+b z$ is not conformally Cotton and thus, not conformally Einstein.

Proof. Recall that a metric which is conformally Einstein is conformally Cotton. This means that there exists a gradient field $T$ such that

$$
C(T):=C+W(T, ., ., .) \equiv 0,
$$

where $W$ is the Weyl tensor and $C$ is the Cotton tensor (see e.g. [17]). Writing $T=\left(\Upsilon^{1}, \ldots, \Upsilon^{5}\right)$ with $\Upsilon^{i}=\theta^{i}(T)$ and using the formulae in the appendix we get $0 \equiv C(T)_{112}=A_{4} \mathrm{e}^{\frac{4 b}{3} x} \Upsilon^{4}$. Our assumption about $a_{4}, a_{5}$, and $a_{6}$ means that $A_{4} \neq \equiv 0$. Thus, $\Upsilon^{4}$ must be zero. Furthermore, $0 \equiv C(T)_{214}=-A_{4} \mathrm{e}^{\frac{4 b}{3} x} \Upsilon^{1}$, which implies $\Upsilon^{1}=0$. Finally, we get $0 \equiv C(T)_{314}=C_{314}=-\frac{\sqrt{3}}{3} A_{4} \mathrm{e}^{2 b x} \neq 0$. This means that with our assumptions about $F$, the metric $g_{F}$ cannot be conformally Cotton, and hence, not conformally Einstein.

Remark 3.5. We observe the remarkable fact that for any $F$ as in (1.3) the Riemann tensor of $\widetilde{g}_{F}$ considered as an endomorphism of $\Lambda^{2} T^{*} \widetilde{M}$ has rank $\leq 4$. Hence, in order to obtain the 14-dimensional group $\mathrm{G}_{2(2)}$ as holonomy group also derivatives of the curvature have to contribute to the holonomy algebra.

Proposition 3.6. For $F=q^{2}+a_{3} p^{3}+a_{2} p^{2}+a_{1} p+a_{0}+b z$ with $a_{3} \neq 0$ the metric $g_{F}$ is the unique Cotton flat metric in $\left[g_{F}\right]$, but $g_{F}$ is not conformally Einstein. 
Proof. The assumptions on $F$ imply that $A_{4} \equiv 0$ and $A_{5} \equiv 0$. By the formulae in the appendix this implies that the Cotton tensor of $g_{F}$ is zero. Now we find the most general vector $T$ such that $C(T)_{j k l}=W_{i j k l} \Upsilon^{i}=0$. The formulae for the Weyl tensor give that

$$
\begin{aligned}
& W_{i 514} \Upsilon^{i}=W_{1514} \Upsilon^{1}=\mathrm{e}^{\frac{4 b}{3} x} A_{3} \Upsilon^{1} \\
& W_{i 115} \Upsilon^{i}=W_{4115} \Upsilon^{4}=-\mathrm{e}^{\frac{4 b}{3} x} A_{3} \Upsilon^{4},
\end{aligned}
$$

which imply that $\Upsilon^{1}=\Upsilon^{4}=0$. Using $\Upsilon^{1}=0$, we get

$$
0=W_{i 414} \Upsilon^{i}=W_{2414} \Upsilon^{2}=\mathrm{e}^{\frac{4 b}{3} x} A_{3} \Upsilon^{2},
$$

and thus $\Upsilon^{2}=0$. Now the condition

$$
0=W_{i 114} \Upsilon^{i}=W_{3114} \Upsilon^{3}+W_{5114} \Upsilon^{5}
$$

gives $\Upsilon^{5}=\frac{2^{4 / 3}}{\sqrt{3}} b \Upsilon^{3}$. This turns out to solve all the remaining equations (3.7). Hence, the most general $T$ solving (3.7) is given by $\Upsilon^{i}=f\left(0,0,1,0, \frac{2^{4 / 3}}{\sqrt{3}} b\right)$ with a smooth function $f$. To define a scale $\Upsilon$ such that $\mathrm{e}^{2 \Upsilon} g_{F}$ is Einstein, this $T$ must be a gradient, which means that $\mathrm{d} \Upsilon=g(T,)=.f\left(\frac{2^{4 / 3}}{\sqrt{3}} b \theta^{1}+\theta^{3}\right)$. Thus in such a case $\mathrm{d}(f \tau)=0$, where $\tau=\left(\frac{2^{4 / 3}}{\sqrt{3}} b \theta^{1}+\theta^{3}\right)$. Calculating $\mathrm{d} \tau$ we get

$$
0=\mathrm{d}(f \tau) \wedge \theta^{1} \wedge \theta^{3}=f \mathrm{~d} \tau \wedge \theta^{1} \wedge \theta^{3}=f \frac{2}{\sqrt{3}} \mathrm{e}^{\frac{2 b}{3} x} \theta^{1} \wedge \theta^{3} \wedge \theta^{4} \wedge \theta^{5} .
$$

But this implies that $f \equiv 0$. Hence, $g_{F}$ is the unique (up to a constant) Cotton flat metric in $\left[g_{F}\right]$. The formulae for $\mathrm{P}$ show that it is not Einstein. Thus, there is no Einstein metric in $\left[g_{F}\right]$.

We can summarise the results about whether $\left[g_{F}\right]$ contains an Einstein metric in

Theorem 3.7. Let $F$ be given by $F=q^{2}+\sum_{i=0}^{6} a_{i} p^{i}+b z$ with at least one of $a_{3}, a_{4}, a_{5}, a_{6}$ not equal to zero. Then the conformal class $\left[g_{F}\right]$ does not contain an Einstein metric. If furthermore $a_{4}=a_{5}=a_{6}=0$, then $g_{F}$ is Cotton flat.

Now we study the property of $\left[g_{F}\right]$ whether it contains a metric $g$ with the properties (2.11) and (2.12), which were subject to Theorem 2.6.

Lemma 3.8. Let $(M, g)$ be pseudo-Riemannian manifold that admits a null line $L$. Then $\operatorname{Ric}(X,)=$.0 for all $X \in L^{\perp}$ if and only if locally there is a vector field $K$ tangent to $L$ and smooth function $\phi$ such that $\mathrm{Ric}=\phi g(K,.) \otimes g(K,$.$) . Each$ of these properties implies that $(M, g)$ has vanishing scalar curvature and thus $\mathrm{P}=\frac{1}{n-2}$ Ric.

Proof. This is easily verified in a basis. 
Lemma 3.9. Let $(M, g)$ be pseudo-Riemannian manifold that admits a $\nabla^{g}$-parallel null line $L$ and satisfies the condition that $\operatorname{Ric}(X,)=$.0 for all $X \in L^{\perp}$. Then the Weyl tensor $W$ of $g$ and hence of every metric in the conformal class of $g$ satisfies

$$
W(., K, K, X)=0 \text {, for all } K \in L \text { and } X \in L^{\perp} .
$$

Proof. Since $L$ is parallel, the curvature $R$ of $g$ satisfies $R(U, V, K, X)=0$ for all $U, V \in T M, K \in L$ and $X \in L^{\perp}$. Then the property $\operatorname{Ric}(X,)=$.0 and hence $\mathrm{P}(X,)=$.0 yields $(3.8)$.

Theorem 3.10. Let $F$ be given by $F=q^{2}+\sum_{i=0}^{6} a_{i} p^{i}+b z$ with at least one of $a_{3}, a_{4}, a_{5}, a_{6}$ not equal to zero. Then the conformal class $\left[g_{F}\right]$ does not contain a metric $g$ with the properties (2.11) and (2.12).

Proof. We consider the most general null line $L$ for $\left[g_{F}\right]$. We will show that there is no metric $g$ in the conformal class $\left[g_{F}\right]$ such that conditions (2.11) and (2.12) hold for $L$. Let $K^{i}$ be tangent to $L$. We have to exclude the following four cases:
a) $K^{i}=\left(1, \alpha, \beta, \gamma, \alpha \gamma-\frac{1}{2} \beta^{2}\right)$,
b) $K^{i}=\left(0,1, \beta, \frac{1}{2} \beta^{2}, \gamma\right)$,
c) $K^{i}=(0,0,0,1, \gamma)$,
d) $K^{i}=(0,0,0,0,1)$,

where $\alpha, \beta$, and $\gamma$ are arbitrary functions. This is achieved by analysing the conformally invariant condition (3.8) and, in cases b) and d), the properties (2.11) and (2.12), i.e. $\hat{\nabla}_{a} K_{b}=f_{a} K_{b}$ and $\hat{P}_{a b}=\Phi K_{a} K_{b}$ for all metrics $\hat{g} \in\left[g_{F}\right]$. The calculations, in which we will refer to the polynomials as defined in (3.3) and (3.4), are based on the formulae provided in the appendix. Recall that $A_{3} \equiv 0$ means that $g_{F}$ is conformally Einstein, and $A_{4} \equiv 0$ means that $g_{F}$ is conformally Cotton and not conformally Einstein if $a_{3} \neq 0$.

c) Case c) is excluded because it is in contradiction with $g_{F}$ not being conformal to Einstein: One of the vectors from $K^{\perp}$ is $X^{i}=(1, \gamma, 0,0,0)$. For this we get that $W_{2 b c d} K^{b} K^{d} X^{c}=A_{3} \mathrm{e}^{\frac{4 b}{3} x}$. Hence, condition (3.8) implies that $g_{F}$ is conformal to an Einstein metric.

a) First we exclude case a) in the not conformally Cotton case, i.e. when at least one of $a_{i} \neq 0$ for $i=4,5,6$, i.e. $A_{4} \not \equiv 0$. In this case equation (3.8) for $X^{i}=\left(1,0,0,0, \frac{1}{2} \beta^{2}-\alpha \gamma\right) \in K^{\perp}$ gives

$$
0=W_{5 b c d} K^{b} K^{d} X^{c}=-A_{3} \gamma \mathrm{e}^{\frac{4 b}{3} x},
$$


and thus $\gamma=0$. This yields

$$
0=W_{1 b c d} K^{b} K^{d} X^{c}=-2 A_{4} \beta^{2} \mathrm{e}^{\frac{4 b}{3} x},
$$

and therefore $\beta=0$. Hence $X^{i}=(1,0,0,0,0)$. This gives

$$
0=W_{4 b c d} K^{b} K^{d} X^{c}=A_{4} \alpha \mathrm{e}^{\frac{4 b}{3} x},
$$

and thus $\alpha=0$, i.e. $K^{i}=(1,0,0,0,0)$. Furthermore, for $Y^{i}=(0,1,0,0,0) \in$ $K^{\perp}$ we get

$$
0=W_{4 b c d} K^{b} K^{d} Y^{c}=-A_{4} \mathrm{e}^{\frac{4 b}{3} x} \neq 0,
$$

which gives the contradiction.

Now we exclude case a) when $g_{F}$ is Cotton flat, i.e. $a_{4}=a_{5}=a_{6}=0$, which means $A_{4} \equiv 0$ and $a_{3} \neq 0$. Equation (3.8) gives

$$
0=W_{5 b c d} K^{b} K^{d} X^{c}=-A_{3} \gamma \mathrm{e}^{\frac{4 b}{3} x}
$$

which implies $\gamma=0$. Furthermore, for $Z^{i}=(0,0,1,0,-\beta) \in K^{\perp}$ we obtain from Equation (3.8) that

$$
W_{4 b c d} K^{b} K^{d} Z^{c}=-\frac{A_{3}}{\sqrt{3} \cdot 2^{2 / 3}} \mathrm{e}^{\frac{4 b}{3} x}\left(4 b+\sqrt{3} \cdot 2^{2 / 3} \beta\right) .
$$

This means that $\beta=-\frac{2^{4 / 3}}{\sqrt{3}} b$. Using this, equation (3.8) for $U^{i}=(0,0,0,1, \alpha) \in$ $K^{\perp}$ gives

$$
0=W_{5 b c d} K^{b} K^{d} U^{c}=A_{3} \mathrm{e}^{\frac{4 b}{3} x},
$$

which is in contradiction with $A_{3} \neq 0$.

b) For case b) equation (3.8) with vector $X^{i}=(1,0,0, \gamma, 0) \in K^{\perp}$ gives

$$
W_{4 b c d} K^{b} K^{d} X^{c}=-\frac{A_{3}}{2} \beta^{2} \mathrm{e}^{\frac{4 b}{3} x}=0,
$$

and thus $\beta=0$. Hence, in this case we have $K^{i}=(0,1,0,0, \gamma)$. Now we calculate $\hat{\nabla} K$ for the metric $\hat{g}=\mathrm{e}^{2 \Upsilon} g$ with an arbitrary function $\Upsilon=$ $\Upsilon(x, y, z, p, q)$. The condition that $K$ is tangent to a parallel null line for some $\Upsilon$ implies that the first component of $\hat{\nabla} K$ must be zero. This implies that $\Upsilon=\Upsilon(x, y, p)$. Using this we find that the third component of $\hat{\nabla} K$ vanishes if and only if

$$
0=\frac{\sqrt{3}}{2^{4 / 3}} \gamma \partial_{p} \Upsilon \theta^{1}-\frac{\sqrt{3}}{3}\left(\gamma+3 \cdot 2^{-4 / 3} \partial_{p} \Upsilon\right) \theta^{4}
$$


This yields $\Upsilon=\Upsilon(x, y)$ and $\gamma=0$. With $\gamma=0$, the fifth component of $\hat{\nabla} K$ must vanish, which implies $\Upsilon=\Upsilon(x)$. Calculating the Schouten tensor $\mathrm{P}$ for such $\Upsilon$ we find that $\hat{\mathrm{P}}_{14}$ vanishes if and only if $A_{3}$ vanishes. Since in the conformally non-Einstein case the quantity $A_{3}$ is non-vanishing, we get a contradiction with the condition $\hat{\mathrm{P}}_{14}=0$ which is implied by $\hat{\mathrm{P}}_{a b}=\phi K_{a} K_{b}$ and the $K$ with $\beta=\gamma=0$. This excludes the case b).

d) A similar argument can be used in the case d). Here $K=(0,0,0,0,1)$ and the most general choice of the metric $\hat{g}=\mathrm{e}^{2 \Upsilon} \mathrm{g}$ leads to the following formula for the second component of the covariant derivative of $K:(\hat{\nabla} K)^{2} \wedge \theta^{1} \wedge \theta^{2}=$ $-\frac{1}{2 \sqrt{3}} \hat{\theta}^{3} \wedge \hat{\theta}^{1} \wedge \hat{\theta}^{2}$. The condition that $K$ is parallel in $\hat{\nabla}$ requires that this must be zero, which excludes the case d) as well.

Hence, for an arbitrary null vector and any metric in the conformal class $\left[g_{F}\right]$ we have shown that conditions (2.11) and (2.12) cannot be satisfied together.

\section{Ambient metrics with holonomy $\mathrm{G}_{2(2)}$}

For those conformal classes introduced in the previous section that are not conformally Einstein the relation between the holonomy of the ambient metric and the conformal holonomy is more involved than in the conformally Einstein case. We will now show that for some $\left[g_{F}\right]$ the ambient metric has holonomy exactly $G_{2(2)}$. The strategy is to show that the ambient manifold admits exactly one parallel spinor which is not null and exclude the existence of holonomy invariant null spaces by using Theorem 3.10.

In Theorem 1.1 in the introduction we have proven that the ambient metric for $g_{F}$ as defined in (1.4) is given as

$$
-2 \mathrm{~d} u \mathrm{~d} t+t^{2} g_{F}-2 t u \mathrm{P}-u^{2} B .
$$

Calculating $\mathrm{P}$ and $B$ explicitly in the appendix this reads as

$$
\begin{aligned}
\tilde{g}_{F}= & -2 \mathrm{~d} t \mathrm{~d} u+t^{2} g_{F}+2 t u \mathrm{e}^{\frac{4 b}{3} x}\left(A_{4}\left(\theta^{1}\right)^{2}+2 A_{3} \theta^{1} \theta^{4}+A_{2} \theta^{4}\right) \\
& +\frac{1}{6} u^{2} \mathrm{e}^{\frac{8 b}{3} x}\left(A_{6} \theta^{1} \theta^{2}+2 A_{5} \theta^{1} \theta^{4}+A_{4}\left(\theta^{4}\right)^{2}\right),
\end{aligned}
$$

where $A_{i}$ 's are defined in (1.5), (1.6), (3.3)-(3.6). Note that the choice of a different $g_{F}$ in the conformal class than in [32] results in a different coordinate system in which the ambient metric is expressed. Note also that in this form the ambient metric for $\left[g_{F}\right]$ has no $u^{2}$ terms if $g_{F}$ is Cotton flat, i.e. if $a_{4}=a_{5}=a_{6}=0$. This means that for such $F$ it truncates at the same order as the ambient metric of a conformal class with an Einstein metric, although it does not contain an Einstein metric if $a_{3} \neq 0$. 
In order to absorb the terms in the ambient metric coming from the terms of first and second order in $u$, we introduce the following co-frame on $M$ :

$$
\begin{aligned}
\eta^{1}= & t \theta^{1} \\
\eta^{2}= & t \theta^{2}-\frac{1}{12} \mathrm{e}^{\frac{4 b}{3} x} \frac{u}{t}\left(12 A_{2} t+A_{4} \mathrm{e}^{\frac{4 b}{3} x}\right) \theta^{4} \\
& +\frac{1}{12} \mathrm{e}^{\frac{4 b}{3} x} \frac{u}{t}\left(-24 A_{3} t+12 \cdot 2^{1 / 3} A_{4} p t-2 A_{5} \mathrm{e}^{\frac{4 b}{3} x} u+2^{1 / 3} A_{6} \mathrm{e}^{\frac{4 b}{3} x} p u\right) \theta^{1} \\
\eta^{3}= & t \theta^{3} \\
\eta^{4}= & t \theta^{4} \\
\eta^{5}= & t \theta^{5}+\frac{1}{12} \mathrm{e}^{\frac{4 b}{3} x} \frac{u}{t}\left(12 A_{4}+A_{6} \mathrm{e}^{\frac{4 b}{3} x} u\right)\left(\theta^{1}+2^{1 / 3} p \theta^{4}\right) .
\end{aligned}
$$

Then we write the ambient metric as

$$
\widetilde{g}_{F}=-2 \mathrm{~d} t \mathrm{~d} u+2 \eta^{1} \eta^{5}-2 \eta^{2} \eta^{4}+\left(\eta^{3}\right)^{2} .
$$

For the calculation of the parallel spinor we use the orthonormal basis

$$
\begin{aligned}
& \xi^{0}=\frac{1}{\sqrt{2}}(\mathrm{~d} t-\mathrm{d} u), \xi^{1}=\frac{1}{\sqrt{2}}\left(\eta^{1}+\eta^{5}\right), \xi^{2}=\frac{1}{\sqrt{2}}\left(\eta^{2}-\eta^{4}\right), \xi^{3}=\eta^{3} \\
& \xi^{4}=\frac{1}{\sqrt{2}}\left(\eta^{2}+\eta^{4}\right), \xi^{5}=\frac{1}{\sqrt{2}}\left(\eta^{1}-\eta^{5}\right), \xi^{6}=\frac{1}{\sqrt{2}}(\mathrm{~d} t+\mathrm{d} u),
\end{aligned}
$$

in which $\widetilde{g}_{F}$ reads as

$$
\widetilde{g}_{F}=\widetilde{g}_{i j} \xi^{i} \xi^{j}=\left(\xi^{0}\right)^{2}+\left(\xi^{1}\right)^{2}+\left(\xi^{2}\right)^{2}+\left(\xi^{3}\right)^{2}-\left(\xi^{4}\right)^{2}-\left(\xi^{5}\right)^{2}-\left(\xi^{6}\right)^{2} .
$$

We represent the Clifford algebra $\mathrm{Cl}(4,3)$ by means of $\sigma$-matrices satisfying the relation

$$
\sigma_{i} \sigma_{j}+\sigma_{j} \sigma_{i}=2 \widetilde{g}_{i j} \mathbb{I}_{8}
$$

They are given as:

$$
\begin{aligned}
\sigma_{0} & =\left(\begin{array}{cc}
0 & \gamma_{0} \\
\gamma_{0} & 0
\end{array}\right), \sigma_{1}=\left(\begin{array}{cc}
0 & \gamma_{2} \\
\gamma_{2} & 0
\end{array}\right), \sigma_{2}=\left(\begin{array}{cc}
0 & \gamma_{4} \\
\gamma_{4} & 0
\end{array}\right) \quad \sigma_{3}=\left(\begin{array}{cc}
\mathbb{I}_{4} & 0 \\
0 & -\mathbb{I}_{4}
\end{array}\right) \\
\sigma_{4} & =\left(\begin{array}{cc}
0 & \gamma_{1} \\
\gamma_{1} & 0
\end{array}\right), \sigma_{5}=\left(\begin{array}{cc}
0 & \gamma_{3} \\
\gamma_{3} & 0
\end{array}\right), \sigma_{6}=\left(\begin{array}{cc}
0 & -\mathbb{I}_{4} \\
\mathbb{I}_{4} & 0
\end{array}\right),
\end{aligned}
$$


where

$$
\begin{aligned}
& \gamma_{0}=\left(\begin{array}{llll}
0 & 0 & 0 & 1 \\
0 & 0 & 1 & 0 \\
0 & 1 & 0 & 0 \\
1 & 0 & 0 & 0
\end{array}\right), \quad \gamma_{2}=\left(\begin{array}{rrrr}
0 & 0 & 1 & 0 \\
0 & 0 & 0 & -1 \\
1 & 0 & 0 & 0 \\
0 & -1 & 0 & 0
\end{array}\right), \gamma_{4}=\left(\begin{array}{rrrrr}
1 & 0 & 0 & 0 \\
0 & 1 & 0 & 0 \\
0 & 0 & -1 & 0 \\
0 & 0 & 0 & -1
\end{array}\right), \\
& \gamma_{1}=\left(\begin{array}{rrrr}
0 & 0 & 0 & -1 \\
0 & 0 & 1 & 0 \\
0 & -1 & 0 & 0 \\
1 & 0 & 0 & 0
\end{array}\right), \gamma_{3}=\left(\begin{array}{rrrr}
0 & 0 & -1 & 0 \\
0 & 0 & 0 & -1 \\
1 & 0 & 0 & 0 \\
0 & 1 & 0 & 0
\end{array}\right) .
\end{aligned}
$$

Note that

$$
\gamma_{i}^{2}=(-1)^{i} \mathbb{I}_{4}
$$

which implies relation (4.2). The invariant scalar product $\langle.,$.$\rangle is given by$

$$
\langle\varphi, \psi\rangle:=-\left(\sigma_{4} \cdot \sigma_{5} \cdot \sigma_{6} \cdot \varphi, \psi\right),
$$

where (., .) is the Euclidean standard scalar product on $\mathbb{R}^{8}$. In the standard basis of $\mathbb{R}^{8}$ the split signature scalar product $\langle.,$.$\rangle is given by the matrix$

$$
\left(\begin{array}{rrrr}
0 & 0 & \mathbb{J}_{2} & 0 \\
0 & 0 & 0 & -\mathbb{J}_{2} \\
-\mathbb{J}_{2} & 0 & 0 & 0 \\
0 & \mathbb{J}_{2} & 0 & 0
\end{array}\right),
$$

where $\mathbb{J}_{2}=\left(\begin{array}{cc}0 & -1 \\ 1 & 0\end{array}\right)$. It satisfies the relation

$$
\left\langle\sigma_{i} \cdot \varphi, \psi\right\rangle=-\left\langle\sigma_{i} \cdot \psi, \varphi\right\rangle,
$$

which implies its invariance. Hence, the scalar product gives a metric on the spin bundle, which we denote by the same symbol, and which is parallel with respect to the lift of the Levi-Civita connection $\widetilde{\nabla}$.

Then we have to solve the parallel spinor equations

$$
0=\widetilde{\nabla} \psi=\mathrm{d} \psi+\frac{1}{4} \sum_{k, l=0}^{6} \widetilde{\Gamma}^{k l} \sigma_{k} \sigma_{l} \psi
$$

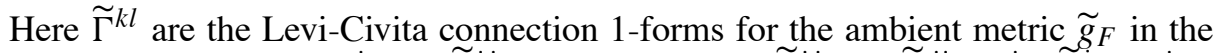
orthonormal co-frame $\xi^{i}$. I.e., $\widetilde{\Gamma}^{i j}$ are determined by $\widetilde{\Gamma}^{i j}=-\widetilde{\Gamma}^{j i}, d \xi^{i}+\widetilde{\Gamma}^{i}{ }_{j} \wedge \xi^{j}=$ 0 , and $\widetilde{\Gamma}^{i j}=\widetilde{\Gamma}_{k}^{i} \tilde{g}^{k j}$.

Proposition 4.1. Let $F=q^{2}+\sum_{i=0}^{6} a_{i} p^{i}+b z$. Then the non-null spinor

$$
\psi=\left(0,-\mathrm{e}^{\frac{b}{3} x}, \mathrm{e}^{\frac{b}{3} x}, 0, \sqrt{\frac{2}{3}} \mathrm{e}^{\frac{b}{3} x}\left(2^{\frac{1}{3}} b \mathrm{e}^{\frac{2 b}{3} x}-3\right), 0,0, \sqrt{\frac{2}{3}} \mathrm{e}^{\frac{b}{3} x}\left(2^{\frac{1}{3}} b \mathrm{e}^{\frac{2 b}{3} x}+3\right)\right)
$$


is a solution of the parallel spinor equation (4.4). In particular, the holonomy of the ambient metric of $\left[g_{F}\right]$ is contained in $\mathrm{G}_{2(2)}$.

Proof. One checks by direct calculations that $\psi$ is parallel and not null with $\langle\psi, \psi\rangle=$ $4 \sqrt{6}$.

For completeness we will give below a formula for the parallel three-form $\omega$ that defines the $\mathrm{G}_{2(2)}$ structure. The form $\omega$ is related to the spinor $\psi$ by the following relation (see for example [23]): First one defines a skew $(2,1)$-tensor $A^{\psi}$ depending on $\psi$ via

$$
X \cdot Y \cdot \psi-\widetilde{g}(X, Y) \psi=A^{\psi}(X, Y) \cdot \psi
$$

and obtains $\omega$ by dualising it

$$
\omega(X, Y, Z):=\widetilde{g}\left(X, A^{\psi}(Y, Z)\right) .
$$

Calculating this with Mathematica we get that $\omega$ is equal to

$$
\begin{aligned}
\omega= & \frac{1}{6 \cdot 2^{5 / 6} \sqrt{3}}\left(18 f(-x)-3 \cdot 2^{1 / 3} f(x)+4 b^{2} f(x)\right)\left(\xi^{012}-\xi^{146}\right) \\
& +\frac{1}{6 \cdot 2^{5 / 6} \sqrt{3}}\left(18 f(-x)+3 \cdot 2^{1 / 3} f(x)-4 b^{2} f(x)\right)\left(\xi^{014}+\xi^{126}\right) \\
& -\frac{1}{6 \cdot 2^{5 / 6} \sqrt{3}}\left(18 f(-x)+3 \cdot 2^{1 / 3} f(x)+4 b^{2} f(x)\right)\left(\xi^{025}-\xi^{456}\right) \\
& +\frac{1}{6 \cdot 2^{5 / 6} \sqrt{3}}\left(-18 f(-x)+3 \cdot 2^{1 / 3} f(x)+4 b^{2} f(x)\right)\left(\xi^{045}+\xi^{256}\right) \\
& -\frac{2^{1 / 6} b\left(3 \cdot 2^{2 / 3}-b f(x)\right)}{\sqrt{3}\left(-3+2^{1 / 3} b f(x)\right)}\left(\xi^{016}+\xi^{124}\right)+\frac{2^{1 / 3 b}}{3} f(x)\left(-\xi^{023}-\xi^{034}+\xi^{236}+\xi^{346}\right) \\
& +\xi^{036}+\xi^{135}+\xi^{234}+\frac{2^{5 / 6} b}{\sqrt{3}}\left(\xi^{056}-\xi^{245}\right)
\end{aligned}
$$

with

$$
f(x):=\mathrm{e}^{\frac{2 b}{3} x}
$$

and $\xi^{i j k}:=\xi^{i} \wedge \xi^{j} \wedge \xi^{k}$, where $\xi^{i}$ is the orthonormal coframe given on page 4. A direct calculation verifies that $\omega$ and its Hodge dual are closed.

In order to conclude the proof of Theorem 1.1 it only remains to prove the following lemma. 
Lemma 4.2. If a 7-dimensional spin manifold $\widetilde{M}$ with metric $\widetilde{g}$ of signature $(4,3)$ admits a parallel non-null spinor $\psi$ and a parallel line of null spinors, then there is a parallel line bundle of tangent vectors on $\widetilde{M}$.

Proof. We fix a spinor $\varphi$ that spans the parallel line of spinors. There is a 1-form $f$ such that $\widetilde{\nabla} \varphi=f \otimes \varphi$. We associate to $\psi$ and $\varphi$ a vector field $V$ via transposing the Clifford multiplication, i.e.

$$
\tilde{g}(V, X)=\langle X \cdot \psi, \varphi\rangle=-\langle X \cdot \varphi, \psi\rangle
$$

for all $X \in T \tilde{M}$. The well known formula

$$
Y(\langle X \cdot \varphi, \psi\rangle)=\left\langle\widetilde{\nabla}_{Y} X \cdot \psi, \varphi\right\rangle+\left\langle X \cdot \widetilde{\nabla}_{Y} \psi, \varphi\right\rangle+\left\langle X \cdot \psi, \widetilde{\nabla}_{Y} \varphi\right\rangle
$$

for two spinor fields $\varphi$ and $\psi$, and two vector fields $X$ and $Y$, shows that $V$ spans a parallel line. Indeed, it implies that

$$
\begin{aligned}
\widetilde{g}\left(\widetilde{\nabla}_{X} V, Y\right) & =X(\widetilde{g}(V, Y))-\widetilde{g}\left(V, \widetilde{\nabla}_{X} Y\right) \\
& =X(\langle Y \cdot \psi, \varphi\rangle)-\left\langle\widetilde{\nabla}_{X} Y \cdot \psi, \varphi\right\rangle \\
& =f(X)\langle Y \cdot \psi, \varphi\rangle \\
& =f(X) \widetilde{g}(V, Y),
\end{aligned}
$$

for all $X, Y \in T \tilde{M}$. For the proof, we have to exclude that $V \equiv 0$, i.e. that

$$
g(V, X)=\langle X \cdot \psi, \varphi\rangle=0
$$

for all $X \in T \widetilde{M}$. We will show that this contradicts $\psi$ being not null and $\varphi$ being null. To this end, at each tangent space $T_{p} \widetilde{M}=\mathbb{R}^{4,3}$, consider the map

$$
\mathbb{R}^{4,3} \ni X \mapsto X \cdot \psi \in \Delta_{4,3} .
$$

Using the transitive action of $\operatorname{Spin}(4,3)$ on spheres in $\mathbb{R}^{4,4}$, one shows [23] that $\psi$ is not null if and only if this map has a trivial kernel. Hence, with $\psi$ being not null, the vector space

$$
W:=\left\{X \cdot \psi \mid X \in \mathbb{R}^{4,3}\right\}
$$

has dimension seven. Furthermore, property (4.3) implies

$$
2\langle X \cdot \psi, Y \cdot \psi\rangle=-g(X, Y)\langle\psi, \psi\rangle,
$$

for all $X, Y \in \mathbb{R}^{4,3}$. Since $\psi$ is not null, this shows that $W \subset \mathbb{R}^{4,4}$ is nondegenerate. Equation (4.5) then implies that $\mathbb{R} \varphi=W^{\perp}$ which contradicts $\varphi$ being null. 
This lemma shows that the existence of a parallel maximal totally null subspace yields the existence of a parallel line bundle in the tangent bundle for the ambient metric, which by Theorem 2.2 contradicts $g_{F}$ not being conformally Einstein.

Remark 4.3. Note also that the existence of two non-null spinor yields the existence of a parallel vector field. This is true by the result in [23] that the isotropy group of two spinors that are not null is given by $\operatorname{SU}(1,2)$ or $\operatorname{SL}(3, \mathbb{R})$. Both cases imply that there is a parallel vector field on $M$ that is not null.

\section{Appendix}

Here we will give formulae for the Levi-Civita connection of $g_{F}$, its Schouten, Weyl, Cotton and Bach tensor. $g_{F}$ is given as in (1.4), with $\theta^{i}$,s as on page 409. In this coframe the Levi-Civita connection 1-forms, i.e. matrix-valued 1-forms satisfying $\mathrm{d} \theta^{\mu}+\Gamma_{\nu}^{\mu} \wedge \theta^{\nu}=0, \Gamma_{\mu \nu}+\Gamma_{\nu \mu}=0, \Gamma_{\mu \nu}=g_{\mu \sigma} \Gamma_{\nu}^{\sigma}$, are:

$$
\begin{aligned}
& \Gamma_{12}=\Gamma_{23}=\Gamma_{25}=0 \\
& \Gamma_{34}=-\frac{2^{1 / 3}}{3} b \hat{\theta}^{3}+\frac{1}{\sqrt{3}} \hat{\theta}^{5} \\
& \Gamma_{35}=-\frac{1}{\sqrt{3}} \hat{\theta}^{4} \\
& \Gamma_{45}=\frac{2^{1 / 3}}{3} b \hat{\theta}^{1}+\frac{1}{2 \sqrt{3}} \hat{\theta}^{3} \\
& \Gamma_{15}=-\frac{2^{1 / 3}}{3} b \hat{\theta}^{4} \\
& \Gamma_{24}=-\frac{2^{1 / 3}}{3} b \hat{\theta}^{4} \\
& \Gamma_{13}=-2 \sqrt{3}\left(A_{3} \hat{\theta}^{1}+A_{2} \hat{\theta}^{4}\right) \\
& \Gamma_{14}=2^{4 / 3}\left(2^{4 / 3} A_{3} q-A_{2} b\right) \hat{\theta}^{1}+\frac{3 \sqrt{3}}{2} A_{2} \hat{\theta}^{3}-\frac{2^{1 / 3}}{3} b \hat{\theta}^{5},
\end{aligned}
$$

where $A_{1}$ and $A_{2}$ are defined in (1.5), (1.6) and (3.3). Then the Schouten tensor is given as

$$
\mathrm{P}=-A_{4}\left(\hat{\theta}^{1}\right)^{2}-2 A_{3} \hat{\theta}^{1} \hat{\theta}^{4}-A_{2}\left(\hat{\theta}^{4}\right)^{2}
$$


with $A_{4}$ defined in (3.4). Let $W_{i j k l}$ be the Weyl tensor and $W_{i j}$ be the 2-forms defined by $W_{i j}=\frac{1}{2} W_{i j k l} \theta^{k} \wedge \theta^{l}$. They are given by

$$
\begin{aligned}
W_{12}= & -A_{4} \hat{\theta}^{1} \wedge \hat{\theta}^{4} \\
W_{13}= & -2 A_{4} \hat{\theta}^{1} \wedge \hat{\theta}^{3}+\frac{2^{4 / 3}}{\sqrt{3}}\left(3 \cdot 2^{1 / 3} A_{4} q-A_{3} b\right) \hat{\theta}^{1} \wedge \hat{\theta}^{4} \\
W_{14}= & -A_{4} \hat{\theta}^{1} \wedge \hat{\theta}^{2}+\frac{2^{4 / 3}}{\sqrt{3}}\left(3 \cdot 2^{1 / 3} A_{4} q-A_{3} b\right) \hat{\theta}^{1} \wedge \hat{\theta}^{3} \\
& +\frac{1}{3}\left(27 A_{2}^{2}-12 \cdot 2^{1 / 3} A_{1} A_{3}-6 \cdot 2^{2 / 3} A_{2} b^{2}+40 A_{3} b q-24 \cdot 2^{1 / 3} A_{4} q^{2}\right) \hat{\theta}^{1} \wedge \hat{\theta}^{4} \\
& +A_{3}\left(\hat{\theta}^{1} \wedge \hat{\theta}^{5}+\hat{\theta}^{2} \wedge \hat{\theta}^{4}\right) \\
W_{15}= & W_{24}=A_{3} \hat{\theta}^{1} \wedge \hat{\theta}^{4} \\
W_{23}= & W_{25}=W_{34}=W_{35}=W_{45}=0 .
\end{aligned}
$$

If $C_{i j k}$ is the Cotton tensor, the 2-forms $C_{i}=\frac{1}{2} C_{i j k} \theta^{j} \wedge \theta^{k}$ are given by

$$
\begin{aligned}
& C_{2}=C_{5}=0 \\
& C_{3}=-\frac{\sqrt{3}}{3} A_{4} \mathrm{e}^{2 b x} \theta^{1} \wedge \theta^{4} \\
& C_{4}=A_{4} \mathrm{e}^{2 b x}\left(-\frac{\sqrt{3}}{3} \theta^{1} \wedge \theta^{3}+\frac{2^{2 / 3}}{3} q \theta^{1} \wedge \theta^{4}\right) \\
& C_{1}=-\frac{\sqrt{3}}{3} A_{5} \mathrm{e}^{2 b x} \theta^{1} \wedge \theta^{3}+\frac{2^{1 / 3}}{3}\left(A_{4} b+2^{4 / 3} A_{5} q\right) \mathrm{e}^{2 b x} \theta^{1} \wedge \theta^{4},
\end{aligned}
$$

where $A_{5}$ is defined in (3.5). Finally, the Bach tensor is given by

$$
B=-\frac{1}{6} \mathrm{e}^{\frac{8 b}{3} x}\left(A_{6} \theta^{1} \theta^{2}+2 A_{5} \theta^{1} \theta^{4}+A_{4}\left(\theta^{4}\right)^{2}\right) .
$$

These formulae enable the reader to calculate the connection coefficients of the truncated ambient metric

$$
\widetilde{g}_{F}=-2 \mathrm{~d} u \mathrm{~d} t+t^{2} g_{F}-2 t u \mathrm{P}-u^{2} B .
$$

\section{References}

[1] J. AlT., "Fefferman Constructions in Conformal Holonomy", PhD thesis, HumboldtUniversity Berlin, 2008. 
[2] S. Armstrong, Definite signature conformal holonomy: a complete classification, J. Geom. Phys. 57 (2007), 2024-2048.

[3] S. ARMSTRONG and T. LEISTNER, Ambient connections realising conformal tractor holonomy, Monatsh. Math. 152 (2007), 265-282.

[4] H. BAUM and I. KATH, Parallel spinors and holonomy groups on pseudo-Riemannian spin manifolds, Ann. Global Anal. Geom. 17 (1999), 1-17.

[5] M. BERGER, Sur les groupes d'holonomie homogène des variétés à connexion affine et des variétés riemanniennes, Bull. Soc. Math. France 83 (1995), 279-330.

[6] R. L. Bryant, Metrics with exceptional holonomy, Ann. of Math. 126 (1987), 525-576.

[7] R. L. BRYANT, Conformal geometry and 3-plane fields on 6-manifolds, In: "Developments of Cartan Geometry and Related Mathematical Problems", volume 1502 of RIMS Symposium Proceedings, 2006, 1-15.

[8] R. L. BRYANT and S. M. SAlamon, On the construction of some complete metrics with exceptional holonomy, Duke Math. J. 58 (1989), 829-850.

[9] A. ČAP and A. R. GovER, Standard tractors and the conformal ambient metric construction, Ann. Global Anal. Geom. 24 (2003), 231-259.

[10] A. ČAP and K. SAGERSCHNIG, On Nurowski's conformal structure associated to a generic rank two distribution in dimension five, J. Geom. Phys. 59 (2009), 901.

[11] E. CARTAN, Les systèmes de Pfaff, à cinq variables et les équations aux dérivées partielles du second ordre, Ann. Sci. École Norm. Sup. (3) 27 (1910), 109-192.

[12] V. Cortés, T. Leistner, L. SchäFer and F. SChulte-Hengesbach, Half-flat structures and special holonomy, Proc. London Math. Soc. 102 (2011), 113-158.

[13] B. Doubrov and J. SlovaK, Inclusions between parabolic geometries, Pure Appl. Math. Q. 6 (2010), Special Issue: In honor of Joseph J. Kohn, Part 1 of 2, 755-780.

[14] C. Fefferman and C. R. Graham, Conformal invariants, Astérisque, (Numero Hors Serie) (1985), The mathematical heritage of Élie Cartan (Lyon, 1984), 95-116.

[15] C. FefFerman and C. R. Graham, "The Ambient Metric", Annals of Mathematics Studies, Vol. 178, Princeton University Presse, Princeton, NJ, 2012, x+113 pp.

[16] A. R. Gover and F. LeITNer, A sub-product construction of Poincaré-Einstein metrics, Internat. J. Math. 20 (2009), 1263-1287.

[17] A. R. GOVER and P. NUROWSKI, Obstructions to conformally Einstein metrics in n dimensions, J. Geom. Phys. 56 (2006), 450-484.

[18] C. R. GRAhAm and T. WILlSE, Parallel tractor extension and ambient metrics of holonomy split $G_{2}$, J. Differential Geom., to appear, arXiv:1109.3504v1

[19] M. HAMMERL and K. SAGERSCHNIG, Conformal structures associated to generic rank 2 distributions on 5-manifolds - characterization and Killing-field decomposition, SIGMA Symmetry Integrability Geom. Methods Appl. 5 (2009), Paper 081, 29 pp.

[20] D. Hilbert, Über den Begriff der Klasse von Differentialgleichungen, Math. Ann. 73 (1912), 95-108.

[21] D. D. Joyce, Compact Riemannian 7-manifolds with holonomy $G_{2}$. I, II, J. Differential Geom. 43 (1996), 291-328, 329-375.

[22] D. D. JOYCE, Compact 8-manifolds with holonomy Spin(7), Invent. Math. 123 (1996), 507-552.

[23] I. KATH, $G_{2(2)}^{*}$-structures on pseudo-Riemannian manifolds, J. Geom. Phys. 27 (1998), $155-177$.

[24] S. Kichenassamy, On a conjecture of Fefferman and Graham, Adv. Math. 184 (2004), 268-288.

[25] W. KopCZYŃski, Pure spinors in odd dimensions, Classical Quantum Gravity 14 (1997), A227-A236.

[26] T. LEISTNER, Conformal holonomy of C-spaces, Ricci-flat, and Lorentzian manifolds, Differential Geom. Appl. 24 (2006), 458-478.

[27] T. LeIstNer and P. NUROWSKI, Conformal pure radiation with parallel rays, Classical Quantum Gravity 29 (2012), 055007. 
[28] T. LeISTNER and P. Nurowski, Ambient metrics for n-dimensional pp-waves, Comm. Math. Phys. 296 (2010), 881-898.

[29] F. LEITNER, Normal conformal Killing forms, 2004. arXiv.org:math/0406316.

[30] F. LeITNER, Conformal Killing forms with normalisation condition, Rend. Circ. Mat. Palermo (2) Suppl., 75 (2005), 279-292.

[31] P. NuRowsKi, Differential equations and conformal structures, J. Geom. Phys. 43 (2005), 327-340.

[32] P. NUROWSKI, Conformal structures with explicit ambient metrics and conformal $G_{2}$ holonomy, In: "Symmetries and Overdetermined Systems of Partial Differential Equations", Vol. 144 of IMA Vol. Math. Appl., Springer, New York, 2008, 515-526.

[33] S. M. SAlamon, "Riemannian Geometry and Holonomy Groups", Vol. 201 of Pitmann Research Lecture Notes, 1989.

[34] H. Wu, On the de Rham decomposition theorem, Illinois J. Math. 8 (1964), 291-311.

School of Mathematical Sciences

University of Adelaide

SA 5005, Australia

thomas.leistner@adelaide.edu.au

Instytut Fizyki Teoretycznej

Uniwersytet Warszawski

ul. Hoża 69, 00-681 Warszawa, Poland

nurowski@fuw.edu.pl 